\title{
Cubic polynomials represented by norm forms
}

\author{
A.J. Irving \\ Mathematical Institute, Oxford
}

\begin{abstract}
We show that for an irreducible cubic $f \in \mathbb{Z}[x]$ and a full norm form $\mathbf{N}\left(x_{1}, \ldots, x_{k}\right)$ for a number field $K / \mathbb{Q}$ satisfying certain hypotheses the variety

$$
f(t)=\mathbf{N}\left(x_{1}, \ldots, x_{k}\right) \neq 0
$$

satisfies the Hasse principle. Our proof uses sieve methods.
\end{abstract}

\section{Introduction}

In this paper we will consider the Diophantine equation

$$
f(t)=\mathbf{N}\left(x_{1}, \ldots, x_{k}\right) \neq 0,
$$

where $f \in \mathbb{Z}[x]$ is a polynomial and $\mathbf{N}\left(x_{1}, \ldots, x_{k}\right)$ is a full norm form for some number field $K / \mathbb{Q}$. Thus, for some basis $\omega_{1}, \ldots, \omega_{k}$ for the degree $k$ extension $K / \mathbb{Q}$, we have

$$
\mathbf{N}\left(x_{1}, \ldots, x_{k}\right)=N_{K / \mathbb{Q}}\left(x_{1} \omega_{1}+\ldots+x_{k} \omega_{k}\right) .
$$

We are interested in describing families of fields $K / \mathbb{Q}$ and polynomials $f$ for which (1) satisfies the Hasse principle. This means that if there is a solution in $\mathbb{Q}_{p}^{k+1}$, for all $p$, and in $\mathbb{R}^{k+1}$ then there is one in $\mathbb{Q}^{k+1}$.

Browning and Heath-Brown, in [2], describe many of the existing results on this problem. They establish that the Hasse principle holds when $f$ is an irreducible polynomial of degree 2 and $K / \mathbb{Q}$ is a quartic extension containing a root of $f$. Their results were extended by Derenthal, Smeets and Wei, [7], who establish that for any quadratic $f$ and any extension $K / \mathbb{Q}$ the Brauer-Manin obstruction is the only obstruction to the Hasse principle.

We are interested in the case that $f$ is an irreducible cubic. Previous work establishes that, when $K / \mathbb{Q}$ has degree 2 or 3 , the only obstruction to the Hasse principle is the BrauerManin obstruction. Specifically, if $[K: \mathbb{Q}]=2$ then (11) defines a Châtelet surface so the result follows by the work of Colliot-Thélène, Sansuc and Swinnerton-Dyer [5], whereas if

\footnotetext{
${ }^{0} 2010$ Mathematics Subject Classification. Primary 14G05; Secondary 11D57, 11N36.
} 
$[K: \mathbb{Q}]=3$ it follows from Colliot-Thélène and Salberger [4]. As far as we know, no case of the Hasse principle has been established when $f$ is an irreducible cubic and $[K: \mathbb{Q}]>3$.

If, instead of being irreducible, $f$ splits completely into linear factors over $\mathbb{Q}$ then the problem is considerably different. A recent result of Browning and Matthiesen [3] establishes that for any such $f$ and any number field $K / \mathbb{Q}$ the Hasse principle holds provided that the Brauer-Manin obstruction is empty.

Our main result is the following, which establishes the Hasse principle for a certain class of fields $K / \mathbb{Q}$, whose degree may be arbitrarily large.

Theorem 1.1. Let $f \in \mathbb{Z}[x]$ be an irreducible cubic and let $K / \mathbb{Q}$ be a number field satisfying the Hasse norm principle. Suppose that there exists a prime $q \geq 7$ such that for all but finitely many unramified primes $p$ with $p \not \equiv 1(\bmod q)$ the prime ideal factorisation of $p$,

$$
(p)=\prod_{i=1}^{r} P_{i},
$$

consists of prime ideals $P_{i}$ of norms $p^{f_{i}}$ with $f_{1}, \ldots, f_{r}$ coprime. In addition, assume that the number field generated by $f$ is not contained in the cyclotomic field $\mathbb{Q}\left(\zeta_{q}\right)$. Then (1) satisfies the Hasse principle.

An example of a field $K / \mathbb{Q}$ satisfying all the conditions of this theorem can be found by adjoining to $\mathbb{Q}$ a root of

$$
x^{q}-2,
$$

for any prime $q \geq 7$. Since $[K: \mathbb{Q}]=q$ is prime, the extension $K / \mathbb{Q}$ satisfies the Hasse norm principle by the work of Bartels [1]. In addition, for any prime $p \not \equiv 1(\bmod q)$ the equation

$$
x^{q}-2 \equiv 0 \quad(\bmod p)
$$

has a root. If we exclude finitely many values of $p$ it then follows that $K$ has an integral ideal of norm $p$. This clearly implies that the degrees $f_{i}$ are coprime. In conclusion, for this choice of $K$ and any $f$ which does not generate a subfield of $\mathbb{Q}\left(\zeta_{q}\right)$, (1) satisfies the Hasse principle.

After various algebraic reductions we will prove Theorem 1.1 using sieve methods. We will show that for an integer to be a norm from $K / \mathbb{Q}$ it is sufficient that it satisfies certain congruences and that it has no prime factors $p \equiv 1(\bmod q)$. We may therefore estimate the number of norms in a set of integers by sieving out these primes. Our sieve problem will have dimension $\frac{2}{q-1}$. For large $q$ this is close to 0 and therefore both the upper and lower bounds coming from the sieve are close to the truth. We will show that for $q \geq 7$ the losses in the sieve are sufficiently small to give us a positive lower bound for the number of rational points on (1). There are many well-known applications of sieves whose dimension is an integer or $\frac{1}{2}$. However we are not aware of any existing work which uses a sieve whose dimension is between 0 and $\frac{1}{2}$. 
It seems very likely that the method of this paper can be adapted to prove weak approximation for the variety (1) provided that it can be shown that weak approximation holds for the equation

$$
N_{K / \mathbb{Q}}(u)=1 .
$$

As part of our proof we will show that $p$-adic conditions, for finitely many primes $p$, can be imposed on the variable $t$. To handle the infinite place our sieve would have to be modified: sieving a more general region instead of $[0, N]^{2}$. This modification would enable us to find a rational solution, $\left(t, x_{1}, \ldots, x_{k}\right)$, to (1) with the variable $t$ sufficiently close to any idelic point. If we define $x \in K$ by

$$
x=x_{1} \omega_{1}+\ldots+x_{k} \omega_{k} .
$$

then, for any $u \in K$ with $N_{K / \mathbb{Q}}(u)=1$, we can write

$$
u x=y_{1} \omega_{1}+\ldots+y_{k} \omega_{k}
$$

and we have

$$
f(t)=\mathbf{N}\left(y_{1}, \ldots, y_{k}\right) .
$$

It could be shown, using our assumption that weak approximation holds for $N_{K / \mathbb{Q}}(u)=1$, that we can choose a $u$ to make $\left(y_{1}, \ldots, y_{k}\right)$ sufficiently close to any idelic point.

We decided to restrict to the case that $q$ is prime to simplify some of the technical details in the sieve. It seems probable that the argument could work for composite $q$, however the condition $q \geq 7$ would have to be changed as our bounds would involve the value of $\varphi(q)$. We use the assumption that $f$ does not generate a subfield of $\mathbb{Q}\left(\zeta_{q}\right)$ to avoid any correlation between the splitting of primes in the number field $K / \mathbb{Q}$ and in the field generated by $f$. This will be made precise in Lemma 4.1. Observe that if $q \equiv 2(\bmod 3)$ then this condition is satisfied for all cubics $f$ as $\mathbb{Q}\left(\zeta_{q}\right)$ cannot contain a subfield of degree 3 .

\section{Acknowledgements}

This work was completed as part of my DPhil, for which I was funded by EPSRC grant $\mathrm{EP} / \mathrm{P} 505666 / 1$. I am very grateful to the EPSRC for funding me and to my supervisor, Roger Heath-Brown, for all his valuable help and advice.

\section{Algebraic Reduction of the Problem}

It does not matter which norm form $\mathbf{N}$ we choose as they are all equivalent under a linear change of variables defined over $\mathbb{Q}$. In particular we may assume that $\mathbf{N} \in \mathbb{Z}\left[x_{1}, \ldots, x_{k}\right]$. As we eventually wish to apply sieve methods we reduce from a problem over $\mathbb{Q}$ to one over $\mathbb{Z}$. We therefore let $f(a, b)$ denote the homogeneous form of $f$ :

$$
f(a, b)=b^{3} f\left(\frac{a}{b}\right)
$$


Lemma 2.1. Suppose there exist integers $a$ and $b$ for which

$$
b, f(a, b) \in N_{K / \mathbb{Q}}\left(K^{*}\right) .
$$

There is then a solution to (1) over $\mathbb{Q}$.

Proof. Clearly $b \neq 0$. We have

$$
f\left(\frac{a}{b}\right)=b^{-3} f(a, b) .
$$

This is a norm from $K$ since both $b$ and $f(a, b)$ are, and the norm map is multiplicative. In addition $f\left(\frac{a}{b}\right) \neq 0$ since $f$ is irreducible.

We know that the Hasse norm principle holds for $K / \mathbb{Q}$. This means that a nonzero $a \in \mathbb{Q}$ is a norm from $K$ if and only if it is a norm from the group of ideles $I_{K}$ :

$$
\mathbb{Q}^{*} \cap N_{K / \mathbb{Q}}\left(I_{K}\right)=N_{K / \mathbb{Q}}\left(K^{*}\right) .
$$

Let $V$ denote the set of all places of $K$. To show that $a \in \mathbb{Q}^{*}$ is a norm from $K$ it is thus sufficient to construct a sequence $\left(x_{v}\right)_{v \in V}$, where $x_{v}$ is a nonzero element of $K_{v}$, with the following two properties:

1. For all non-Archimedean places $v$, with finitely many exceptions, we have $x_{v} \in R_{v}^{*}$, where $R_{v}$ is the valuation ring of $K_{v}$. This condition ensures that $\left(x_{v}\right) \in I_{K}$.

2. For all places $w$ of $\mathbb{Q}$ we have

$$
\prod_{v \mid w} N_{K_{v} / \mathbb{Q}_{w}}\left(x_{v}\right)=a
$$

We will derive arithmetic conditions which are sufficient to show that a nonzero integer $a$ is in $N_{K / \mathbb{Q}}\left(K^{*}\right)$.

Lemma 2.2. Suppose $a \neq 0$ is an integer. Let $p$ be a prime which does not divide a and which is unramified in $K / \mathbb{Q}$. Then there exist elements $x_{v} \in R_{v}^{*}$, for each place $v$ above $p$, such that

$$
\prod_{v \mid p} N_{K_{v} / \mathbb{Q}_{p}}\left(x_{v}\right)=a .
$$

Proof. Let $v_{1}$ be one of the places above $p$. Since $p$ is unramified in $K / \mathbb{Q}$ we know that the extension $K_{v_{1}} / \mathbb{Q}_{p}$ is unramified. Furthermore, $p \nmid a$ so $a \in \mathbb{Z}_{p}^{*}$. It follows by local class field theory, (for example Gras [9, Corollary 1.4.3, part (ii), page 75]), that there exists $x_{v_{1}} \in K_{v_{1}}$ with

$$
N_{K_{v_{1}} / \mathbb{Q}_{p}}\left(x_{v_{1}}\right)=a .
$$

We must have $x_{v_{1}} \in R_{v_{1}}^{*}$ since

$$
\left|x_{v_{1}}\right|=\left|N_{K_{v_{1}} / \mathbb{Q}_{p}}\left(x_{v_{1}}\right)\right|^{1 /\left[K_{v_{1}}: \mathbb{Q}_{p}\right]}=|a|^{1 /\left[K_{v_{1}}: \mathbb{Q}_{p}\right]}=1 .
$$


For all $v \mid p$ with $v \neq v_{1}$ we define $x_{v}=1$ so

$$
N_{K_{v} / \mathbb{Q}_{p}}\left(x_{v}\right)=1 .
$$

The result follows.

For any fixed $a$ this lemma has dealt with all but a finite number of places. It follows that for the remaining places we need not consider the condition $x_{v} \in R_{v}^{*}$.

Lemma 2.3. Suppose $a \neq 0$ is an integer. Let $p$ be a prime dividing a which is unramified in $K / \mathbb{Q}$. In addition suppose that in the prime ideal factorisation

$$
(p)=\prod P_{i},
$$

with $N\left(P_{i}\right)=p^{f_{i}}$, the various $f_{i}$ are coprime. It follows that there exist $x_{v} \in K_{v}^{*}$, for each $v \mid p$, with

$$
\prod_{v \mid p} N_{K_{v} / \mathbb{Q}_{p}}\left(x_{v}\right)=a .
$$

Proof. Let $v_{i}$ be the place corresponding to the prime ideal $P_{i}$ in the factorisation of $(p)$. We have $\left[K_{v_{i}}: \mathbb{Q}_{p}\right]=f_{i}$ so

$$
N_{K_{v_{i}} / \mathbb{Q}_{p}}(a)=a^{f_{i}} .
$$

Since the $f_{i}$ are coprime there exist integers $k_{i}$ such that

$$
\sum k_{i} f_{i}=1 .
$$

The result follows on taking $x_{v_{i}}=a^{k_{i}}$.

It remains to deal with the primes $p$ which ramify in $K / \mathbb{Q}$. For such primes it is easier to interpret the idelic condition in terms of the solubility of the norm equation over $\mathbb{Q}_{p}$.

Lemma 2.4. Let $a \neq 0$ be an integer. Suppose there exists $x_{1}, \ldots, x_{k} \in \mathbb{Q}_{p}$ such that

$$
a=\mathbf{N}\left(x_{1}, \ldots, x_{k}\right) .
$$

Then there exist $x_{v} \in K_{v}^{*}$, for $v \mid p$, such that

$$
\prod_{v \mid p} N_{K_{v} / \mathbb{Q}_{p}}\left(x_{v}\right)=a .
$$

Proof. Since $a \neq 0$ we know that

$$
\left(x_{1}, \ldots, x_{k}\right) \neq 0 .
$$

Let $x^{(n)}$ be a sequence in $\mathbb{Q}^{k}$ which converges $p$-adically to $\left(x_{1}, \ldots, x_{k}\right)$. Let $\omega_{1}, \ldots, \omega_{k}$ be the basis of $K / \mathbb{Q}$ used to construct the norm form $\mathbf{N}$ and define $y^{(n)} \in K$ by

$$
y^{(n)}=x_{1}^{(n)} \omega_{1}+\ldots+x_{k}^{(n)} \omega_{k} .
$$


For each $v \mid p$ write $y_{v}^{(n)}$ for the image of $y^{(n)}$ under the embedding of $K$ into $K_{v}$. The sequence $y_{v}^{(n)}$ converges to some $x_{v} \in K_{v}^{*}$.

We now have

$$
\prod_{v \mid p} N_{K_{v} / \mathbb{Q}_{p}}\left(x_{v}\right)=\lim _{n \rightarrow \infty} \prod_{v \mid p} N_{K_{v} / \mathbb{Q}_{p}}\left(y_{v}^{(n)}\right) .
$$

However, since $y^{(n)} \in K$ it follows, (for example by Gras [9, Proposition 2.2, page 93]), that

$$
\prod_{v \mid p} N_{K_{v} / \mathbb{Q}_{p}}\left(y_{v}^{(n)}\right)=N_{K / \mathbb{Q}}\left(y^{(n)}\right)=\mathbf{N}\left(x_{1}^{(n)}, \ldots, x_{k}^{(n)}\right) .
$$

We conclude, by continuity of $\mathbf{N}$, that

$$
\prod_{v \mid p} N_{K_{v} / \mathbb{Q}_{p}}\left(x_{v}\right)=\mathbf{N}\left(x_{1}, \ldots, x_{k}\right)=a
$$

Lemma 2.5. Let $p$ be a prime for which (1) has a solution in $\mathbb{Q}_{p}$. There exist $a_{0}, b_{0} \in \mathbb{Z}$ and $l \in \mathbb{N}$, all depending on $p$, satisfying

$$
b_{0}, f\left(a_{0}, b_{0}\right) \neq 0 \quad\left(\bmod p^{l}\right),
$$

such that for any $a, b \in \mathbb{Z}$ with

$$
a \equiv a_{0} \quad\left(\bmod p^{l}\right) \text { and } b \equiv b_{0} \quad\left(\bmod p^{l}\right)
$$

we have

$$
b, f(a, b) \in \mathbf{N}\left(\mathbb{Q}_{p}^{k}\right) \backslash\{0\} .
$$

Proof. For the duration of this proof let

$$
N=\mathbf{N}\left(\mathbb{Q}_{p}^{k}\right) \backslash\{0\}
$$

By assumption there exist $a_{1}, b_{1} \in \mathbb{Z}_{p}$ with $b_{1} \neq 0$ such that

$$
f\left(\frac{a_{1}}{b_{1}}\right) \in N
$$

By replacing $\left(a_{1}, b_{1}\right)$ by $\left(b_{1}^{k-1} a_{1}, b_{1}^{k}\right)$ we may assume that $b_{1} \in N$ and therefore $f\left(a_{1}, b_{1}\right) \in N$. The set $N$ is open and $f$ is continuous with respect to the $p$-adic topology. It follows that there exists $\delta>0$ such that for any $a, b \in \mathbb{Z}_{p}$ with

$$
\left|a-a_{1}\right|,\left|b-b_{1}\right|<\delta
$$

we have

$$
b, f(a, b) \in N
$$


For an $l \in \mathbb{N}$ which is sufficiently large in terms of $\delta$ the hypotheses (21) are equivalent to

$$
a \equiv a_{1} \quad\left(\bmod p^{l}\right), \quad b \equiv b_{1} \quad\left(\bmod p^{l}\right) .
$$

The result follows on taking $a_{0}, b_{0} \in \mathbb{Z}$ which are congruent modulo $p^{l}$ to $a_{1}, b_{1}$. Since

$$
b_{1}, f\left(a_{1}, b_{1}\right) \neq 0
$$

we can guarantee that

$$
b_{0}, f\left(a_{0}, b_{0}\right) \neq 0 \quad\left(\bmod p^{l}\right)
$$

provided $l$ is large enough.

We may now use all the previous lemmas to reduce our original problem to one involving prime divisors of $b$ and $f(a, b)$.

Lemma 2.6. Suppose that (11) has solutions in every $\mathbb{Q}_{p}$ and in $\mathbb{R}$. Let $\mathcal{P}_{1}$ be a finite set of primes which contains the ramified primes in $K / \mathbb{Q}$ as well as the finitely many primes $p \not \equiv 1$ $(\bmod q)$ for which the degrees $f_{i}$ are not coprime. Then there exists $a \Delta \in \mathbb{N}$, divisible only by primes in $\mathcal{P}_{1}$, and integers $a_{0}, b_{0}$ such that if $p \in \mathcal{P}_{1}$ and $p^{l}$ is the maximal power of $p$ dividing $\Delta$ then

$$
b_{0}, f\left(a_{0}, b_{0}\right) \not \equiv 0 \quad\left(\bmod p^{l}\right)
$$

and the following implication is true.

Suppose that $a, b$ are integers for which the following hold:

1. We have

$$
a \equiv a_{0} \quad(\bmod \Delta) \text { and } b \equiv b_{0} \quad(\bmod \Delta) .
$$

2. Each prime $p$ with $p \mid b f(a, b)$ and $p \notin \mathcal{P}_{1}$ satisfies

$$
p \not \equiv 1 \quad(\bmod q) .
$$

3. We have $b \geq 0$ and $f(a, b) \geq 0$.

Then (1) has a solution over $\mathbb{Q}$.

Proof. By Lemma 2.1 it is sufficient to show that

$$
b, f(a, b) \in N_{K / \mathbb{Q}}\left(K^{*}\right) .
$$

By the Hasse norm principle this is equivalent to showing that

$$
b, f(a, b) \in N_{K / \mathbb{Q}}\left(I_{K}\right) .
$$

We must therefore show, for all places of $\mathbb{Q}$, that $b$ and $f(a, b)$ are products of local norms. 
1. For each prime $p \in \mathcal{P}_{1}$ we may use Lemma 2.5 to construct $l_{p}, a_{0, p}, a_{1, p}$. These will satisfy

$$
b_{0, p}, f\left(a_{0, p}, b_{0, p}\right) \not \equiv 0 \quad\left(\bmod p^{l_{p}}\right) .
$$

We now let

$$
\Delta=\prod_{p \in \mathcal{P}_{1}} p^{l_{p}}
$$

and use the Chinese remainder theorem to construct $a_{0}, b_{0}$ satisfying

$$
a_{0} \equiv a_{0, p} \quad\left(\bmod p^{l_{p}}\right), \quad b_{0} \equiv b_{0, p} \quad\left(\bmod p^{l_{p}}\right)
$$

for all $p \in \mathcal{P}_{1}$. It follows by our assumption (3) and Lemma 2.5 that

$$
b, f(a, b) \in \mathbf{N}\left(\mathbb{Q}_{p}^{k}\right) \backslash\{0\} .
$$

We conclude, using Lemma 2.4, that $b$ and $f(a, b)$ are suitable products of local norms for all primes in $\mathcal{P}_{1}$.

2. For primes not in $\mathcal{P}_{1}$ we know that either $p \nmid b$, in which case we use Lemma 2.2 to write $b$ as a suitable product of local norms, or $p \mid b$. In the latter situation $p \not \equiv 1(\bmod q)$ and therefore the degrees of the prime ideals above $p$ are coprime. The required local condition for $b$ now follows by Lemma 2.3. We may use an identical argument for $f(a, b)$.

3. Finally we consider the infinite place. Since $b, f(a, b) \geq 0$ they are both local norms at infinity.

The above cases cover all the places of $\mathbb{Q}$ so the result follows.

For the remainder of this paper we let $\mathcal{P}_{1}$ be a finite set of primes including those which are ramified in $K / \mathbb{Q}$ or which divide the coefficients of $a^{3}$ or $b^{3}$ in the polynomial $f(a, b)$ or which divide the discriminant of $f$. In addition $\mathcal{P}_{1}$ will contain those primes $p \not \equiv 1(\bmod q)$ for which the degrees $f_{i}$ are not coprime. We also include in $\mathcal{P}_{1}$ the prime $q$ and all primes up to some absolute constant $P_{1}$, (which will be determined in Lemma 6.2 below). We let $a_{0}, b_{0}, \Delta$ be the quantities constructed in the last lemma and use the notation $C(a, b)$ to denote that $a, b$ satisfy (3).

Since $f$ is a cubic, we can, without loss of generality, apply a linear change of variable over $\mathbb{Q}$ to guarantee that its leading coefficient is positive and all its real roots are negative. We may thus assume that if $x>0$ then $f(x)>0$. In particular, if $a, b>0$ then $f(a, b)>0$. For a large $N$ we will apply a sieve to count pairs $(a, b) \in(0, N]^{2}$ satisfying $C(a, b)$ for which $b f(a, b)$ has no prime factor $p \notin \mathcal{P}_{1}$ with $p \equiv 1(\bmod q)$. If we can prove a positive lower bound for this quantity then it follows by the last lemma that (1) has a solution over $\mathbb{Q}$. 


\section{Levels of Distribution}

We need various level of distribution results for the values $b f(a, b)$. All implied constants in this section may depend on the polynomial $f$ and on $\Delta$.

The main result of this section, Lemma 3.4, is proved using very similar methods to those of Daniel, [6, Lemmas 3.2 and 3.3]. We extend his results to handle the form $b f(a, b)$, rather than $f(a, b)$, with $a, b$ in a fixed arithmetic progression. Let $\mathcal{R}$ be a compact region of $\mathbb{R}^{2}$. We begin by considering the quantity

$$
R^{*}\left(\mathcal{R}, d_{1}, d_{2}\right)=\#\left\{(a, b) \in \mathcal{R}: C(a, b),(a ; b ; d)=1, d_{1}\left|f(a, b), d_{2}\right| b f(a, b)\right\} .
$$

Throughout this paper we are using $(a ; b)$ to denote the greatest common factor. We need only consider $R^{*}\left(\mathcal{R}, d_{1}, d_{2}\right)$ for $d_{1}, d_{2} \in \mathbb{N}$ satisfying

$$
\left(d_{1} ; d_{2}\right)=\left(d_{1} d_{2} ; \Delta\right)=1
$$

We will write $d=d_{1} d_{2}$.

We say that points $\left(a_{1}, b_{1}\right),\left(a_{2}, b_{2}\right)$ with

$$
\left(a_{1} ; b_{1} ; d\right)=\left(a_{2} ; b_{2} ; d\right)=1
$$

are equivalent modulo $d$ if

$$
\left(a_{1}, b_{1}\right) \equiv \lambda\left(a_{2}, b_{2}\right) \quad(\bmod d)
$$

for some $\lambda \in \mathbb{Z}$ which must necessarily satisfy $(\lambda ; d)=1$. The restriction to points with $(a ; b ; d)=1$ is required to make our notion of equivalence into a valid equivalence relation, (since $(\lambda ; d)=1$ it has an inverse $\bmod d$ ). We will call points with $(a ; b ; d)=1$ primitive modulo $d$. The number of primitive points in each equivalence class which are distinct modulo $d$ is $\varphi(d)$.

Observe that the properties $f(a, b) \equiv 0\left(\bmod d_{1}\right)$ and $b f(a, b) \equiv 0\left(\bmod d_{2}\right)$ are preserved by equivalence. We may therefore define $\mathcal{U}\left(d_{1}, d_{2}\right)$ to be the set of equivalence classes of primitive points modulo $d=d_{1} d_{2}$ for which $f(a, b) \equiv 0\left(\bmod d_{1}\right)$ and $b f(a, b) \equiv 0\left(\bmod d_{2}\right)$.

For an equivalence class $x(\bmod d)$ we let $\lambda(x)$ be the lattice in $\mathbb{Z}^{2}$ generated by the points of $x$. Thus $y \in \lambda(x)$ if and only if there exists some $(a, b) \in x$ and $\lambda \in \mathbb{Z}$ with

$$
y \equiv \lambda(a, b) \quad(\bmod d)
$$


In particular the primitive points in $\lambda(x)$ are precisely those in $x$. It follows that

$$
\begin{aligned}
R^{*}\left(\mathcal{R}, d_{1}, d_{2}\right) & =\sum_{x \in \mathcal{U}\left(d_{1}, d_{2}\right)} \#\{(a, b) \in \mathcal{R} \cap x: C(a, b)\} \\
& =\sum_{x \in \mathcal{U}\left(d_{1}, d_{2}\right)} \#\{(a, b) \in \mathcal{R} \cap \lambda(x): C(a, b),(a ; b ; d)=1\} \\
& =\sum_{x \in \mathcal{U}\left(d_{1}, d_{2}\right)} \sum_{\substack{(a, b) \in \mathcal{R} \cap \lambda(x) \\
C(a, b)}} \sum_{e \mid(a ; b ; d)} \mu(e) \\
& =\sum_{x \in \mathcal{U}\left(d_{1}, d_{2}\right)} \sum_{e \mid d} \mu(e) \#\{(a, b) \in \mathcal{R} \cap \lambda(x): C(a, b), e \mid(a, b)\} \\
& =\sum_{x \in \mathcal{U}\left(d_{1}, d_{2}\right)} \sum_{e \mid d} \mu(e) \#\{(a, b) \in \mathcal{R} \cap \lambda(x, e): C(a, b)\}
\end{aligned}
$$

where $\lambda(x, e)$ is the sublattice of $\lambda(x)$ consisting of points divisible by $e$.

We have $(d ; \Delta)=1$ so $(e ; \Delta)=1$. It follows that the sublattice of $\lambda(x, e)$ consisting of those points which are divisible by $\Delta$ is precisely $\lambda(x, e \Delta)$. It is then clear that the set

$$
\{(a, b) \in \lambda(x, e): C(a, b)\}
$$

is a coset of the lattice $\lambda(x, e \Delta)$.

Lemma 3.1. We have

$$
|\operatorname{det} \lambda(x, e \Delta)|=d e \Delta^{2}
$$

Proof. In general, if a lattice in $\mathbb{Z}^{2}$ is formed from all points whose reduction mod $n$ is in a set of $c$ equivalence classes then its determinant is $\frac{n^{2}}{c}$.

For our specific problem we take $n=d \Delta$. Let $(a, b)$ be a fixed point of $x$. Since $(a ; b ; d)=1$ we know that the number of points modulo $d$ which are multiples of $(a, b)$ and divisible by $e$ is $\frac{d}{e}$. Since $(d ; \Delta)=1$ it follows by the Chinese remainder theorem that $c=\frac{d}{e}$ and therefore

$$
|\operatorname{det} \lambda(x, e \Delta)|=\frac{d^{2} \Delta^{2}}{d / e}=d e \Delta^{2} .
$$

Let $R_{1}(x, e \Delta)$ denote the length of the shortest nonzero vector in $\lambda(x, e \Delta)$. It is clear that this is bounded below by $R_{1}(x)$, the length of the shortest nonzero vector in $\lambda(x)$. Let $V(\mathcal{R})$ and $P(\mathcal{R})$ denote the volume and perimeter of $\mathcal{R}$, respectively. By the standard method for counting lattice points we get

$$
R^{*}\left(\mathcal{R}, d_{1}, d_{2}\right)=\sum_{x \in \mathcal{U}\left(d_{1}, d_{2}\right)} \sum_{e \mid d} \mu(e)\left(\frac{V(\mathcal{R})}{d e \Delta^{2}}+O\left(1+\frac{P(\mathcal{R})}{R_{1}(x)}\right)\right) .
$$


Let $\rho^{*}\left(d_{1}, d_{2}\right)$ denote the number of primitive solutions modulo $d$ to $f(a, b) \equiv 0\left(\bmod d_{1}\right)$ and $b f(a, b) \equiv 0\left(\bmod d_{2}\right)$. Since the number of distinct points modulo $d$ in each equivalence class is $\varphi(d)$ we have

$$
\sum_{x \in \mathcal{U}\left(d_{1}, d_{2}\right)} \sum_{e \mid d} \frac{\mu(e)}{e}=\sum_{x \in \mathcal{U}\left(d_{1}, d_{2}\right)} \frac{\varphi(d)}{d}=\frac{\rho^{*}\left(d_{1}, d_{2}\right)}{d} .
$$

We conclude that for any $\epsilon>0$ we have

$$
R^{*}\left(\mathcal{R}, d_{1}, d_{2}\right)=\frac{\rho^{*}\left(d_{1}, d_{2}\right) V(\mathcal{R})}{d^{2} \Delta^{2}}+O_{\epsilon}\left(d^{\epsilon}\left(1+P(\mathcal{R}) \sum_{x \in \mathcal{U}\left(d_{1}, d_{2}\right)} R_{1}(x)^{-1}\right)\right) .
$$

Averaging this over $d_{1}$ and $d_{2}$ we get

$$
\begin{aligned}
\sum_{\substack{d_{1} \leq D_{1}, d_{2} \leq D_{2} \\
\left(d_{1} ; d_{2}\right)=\left(d_{1} d_{2} ; \Delta\right)=1}} \max _{P(\mathcal{R}) \leq M}\left|R^{*}\left(\mathcal{R}, d_{1}, d_{2}\right)-\frac{\rho^{*}\left(d_{1}, d_{2}\right) V(\mathcal{R})}{d_{1}^{2} d_{2}^{2} \Delta^{2}}\right| \\
\ll_{\epsilon}\left(D_{1} D_{2}\right)^{\epsilon}\left(D_{1} D_{2}+M \sum_{\begin{array}{c}
d_{1} \leq D_{1}, d_{2} \leq D_{2} \\
\left(d_{1} ; d_{2}\right)=\left(d_{1} d_{2} ; \Delta\right)=1
\end{array}} \sum_{x \in \mathcal{U}\left(d_{1}, d_{2}\right)} R_{1}(x)^{-1}\right) .
\end{aligned}
$$

Let $v_{1}(x)$ denote the shortest nonzero vector in $\lambda(x)$. We know that

$$
\left\|v_{1}(x)\right\|^{2} \ll|\operatorname{det} \lambda(x)| \ll D_{1} D_{2} .
$$

We may thus write the final sum as

$$
\sum_{0<a^{2}+b^{2} \ll D_{1} D_{2}} \frac{1}{\sqrt{a^{2}+b^{2}}} \#\left\{d_{1}, d_{2}, x:\left(d_{1} ; d_{2}\right)=\left(d_{1} d_{2} ; \Delta\right)=1, v_{1}(x)=(a, b)\right\} .
$$

If $v_{1}(x)=(a, b)$ then

$$
d_{1} d_{2} \mid b f(a, b) .
$$

We first consider the contribution to the above sum from pairs $(a, b)$ with $b \neq 0$. Since $f$ is irreducible we have $b f(a, b) \neq 0$. It follows that the number of $d_{1}, d_{2}$ for a given $(a, b)$ is bounded by

$$
\tau_{3}(b f(a, b)) \ll_{\epsilon}\left(D_{1} D_{2}\right)^{\epsilon} .
$$

For each such $d_{1}, d_{2}$ the number of $x \in \mathcal{U}\left(d_{1}, d_{2}\right)$ for which $v_{1}(x)=(a, b)$ is at most

$$
\# \mathcal{U}\left(d_{1}, d_{2}\right)=\frac{\rho^{*}\left(d_{1}, d_{2}\right)}{\varphi\left(d_{1} d_{2}\right)} \ll_{\epsilon}\left(D_{1} D_{2}\right)^{\epsilon} .
$$


We conclude that

$$
\begin{aligned}
& \sum_{\substack{0<a^{2}+b^{2} \ll D_{1} D_{2} \\
b \neq 0}} \frac{1}{\sqrt{a^{2}+b^{2}}} \#\left\{d_{1}, d_{2}, x:\left(d_{1} ; d_{2}\right)=\left(d_{1} d_{2} ; \Delta\right)=1, v_{1}(x)=(a, b)\right\} \\
& \ll_{\epsilon}\left(D_{1} D_{2}\right)^{\epsilon} \sum_{\substack{0<a^{2}+b^{2} \ll D_{1} D_{2}\\
}} \frac{1}{\sqrt{a^{2}+b^{2}}} \\
& \ll_{\epsilon}\left(D_{1} D_{2}\right)^{\frac{1}{2}+\epsilon} .
\end{aligned}
$$

It remains to estimate the contribution from pairs $(a, 0)$ :

$$
\sum_{0<a \ll \sqrt{D_{1} D_{2}}} \frac{1}{a} \#\left\{d_{1}, d_{2}, x:\left(d_{1} ; d_{2}\right)=\left(d_{1} d_{2} ; \Delta\right)=1, v_{1}(x)=(a, 0)\right\} .
$$

Suppose that $v_{1}(x)=(a, 0)$. We then have

$$
f(a, 0) \equiv 0 \quad\left(\bmod d_{1}\right) .
$$

Since $f(a, 0) \neq 0$ it follows that the number of possible $d_{1}$ is bounded by

$$
\tau(f(a, 0)) \ll D_{1}^{\epsilon} .
$$

For each such $d_{1}$ the number of $d_{2}$ is clearly bounded by $D_{2}$. As above, the number of $x$ is then $O_{\epsilon}\left(\left(D_{1} D_{2}\right)^{\epsilon}\right)$. We conclude that

$$
\begin{array}{r}
\sum_{0<a \ll \sqrt{D_{1} D_{2}}} \frac{1}{a} \#\left\{d_{1}, d_{2}, x:\left(d_{1} ; d_{2}\right)=\left(d_{1} d_{2} ; \Delta\right)=1, v_{1}(x)=(a, 0)\right\} \\
\ll_{\epsilon} D_{1}^{\epsilon} D_{2}^{1+\epsilon} \sum_{0<a \ll \sqrt{D_{1} D_{2}}} \frac{1}{a} \ll_{\epsilon} D_{1}^{\epsilon} D_{2}^{1+\epsilon} .
\end{array}
$$

Combining these two cases we get

$$
\begin{aligned}
& \sum_{0<a^{2}+b^{2} \ll D_{1} D_{2}} \frac{1}{\sqrt{a^{2}+b^{2}}} \#\left\{d_{1}, d_{2}, x:\left(d_{1} ; d_{2}\right)=\left(d_{1} d_{2} ; \Delta\right)=1, v_{1}(x)=(a, b)\right\} \\
& \ll_{\epsilon}\left(D_{1} D_{2}\right)^{\epsilon}\left(\left(D_{1} D_{2}\right)^{\frac{1}{2}}+D_{2}\right) .
\end{aligned}
$$

We have therefore proved the following.

Lemma 3.2. For any $D_{1}, D_{2}>0$ and any $\epsilon>0$ we have

$$
\begin{gathered}
\sum_{\substack{d_{1} \leq D_{1}, d_{2} \leq D_{2} \\
\left(d_{1} ; d_{2}\right)=\left(d_{1} d_{2} ; \Delta\right)=1}} \max _{P(\mathcal{R}) \leq M}\left|R^{*}\left(\mathcal{R}, d_{1}, d_{2}\right)-\frac{\rho^{*}\left(d_{1}, d_{2}\right) V(\mathcal{R})}{d_{1}^{2} d_{2}^{2} \Delta^{2}}\right| \\
\ll_{\epsilon}\left(D_{1} D_{2}\right)^{\epsilon}\left(D_{1} D_{2}+M\left(\left(D_{1} D_{2}\right)^{\frac{1}{2}}+D_{2}\right)\right) .
\end{gathered}
$$


We next remove the restriction to primitive points. As in Daniel's work, [6, Lemma 3.3], we need the multiplicative functions $\psi_{k}$ which map the prime power $p^{\alpha k+\beta}$, for $1 \leq \beta \leq k$, to $p^{\alpha+1}$.

Let

$$
\begin{aligned}
R\left(\mathcal{R}, d_{1}, d_{2}\right) & =\#\left\{(a, b) \in \mathcal{R}: C(a, b), d_{1}\left|f(a, b), d_{2}\right| b f(a, b)\right\} \\
& =\sum_{\substack{e_{1}\left|\psi_{3}\left(d_{1}\right) \\
e_{2}\right| \psi_{4}\left(d_{2}\right)}} N\left(d_{1}, d_{2}, e_{1}, e_{2}\right)
\end{aligned}
$$

where

$$
\begin{aligned}
& N\left(d_{1}, d_{2}, e_{1}, e_{2}\right) \\
& =\#\left\{(a, b) \in \mathcal{R}: C(a, b),\left(a ; b ; \psi_{3}\left(d_{1}\right) \psi_{4}\left(d_{2}\right)\right)=e_{1} e_{2}, d_{1}\left|f(a, b), d_{2}\right| b f(a, b)\right\} \\
& =\#\left\{(a, b) \in \mathcal{R} / e_{1} e_{2}: C\left(e_{1} e_{2}(a, b)\right),\left(a ; b ; \frac{\psi_{3}\left(d_{1}\right) \psi_{4}\left(d_{2}\right)}{e_{1} e_{2}}\right)=1,\right. \\
& \left.\frac{d_{1}}{\left(d_{1} ; e_{1}^{3}\right)}\left|f(a, b), \frac{d_{2}}{\left(d_{2} ; e_{2}^{4}\right)}\right| b f(a, b)\right\} \\
& =\#\left\{(a, b) \in \mathcal{R} / e_{1} e_{2}: C\left(e_{1} e_{2}(a, b)\right),\left(a ; b ; \frac{d_{1} d_{2}}{\left(d_{1} ; e_{1}^{3}\right)\left(d_{2} ; e_{2}^{4}\right)}\right)=1,\right. \\
& \left.\frac{d_{1}}{\left(d_{1} ; e_{1}^{3}\right)}\left|f(a, b), \frac{d_{2}}{\left(d_{2} ; e_{2}^{4}\right)}\right| b f(a, b)\right\} \\
& =R^{*}\left(\mathcal{R} / e_{1} e, \frac{d_{1}}{\left(d_{1} ; e_{1}^{3}\right)}, \frac{d_{2}}{\left(d_{2} ; e_{2}^{4}\right)} ; e_{1}, e_{2}\right) .
\end{aligned}
$$

Thus

$$
R\left(\mathcal{R}, d_{1}, d_{2}\right)=\sum_{\substack{e_{1}\left|\psi_{3}\left(d_{1}\right) \\ e_{2}\right| \psi_{4}\left(d_{2}\right)}} R^{*}\left(\mathcal{R} / e_{1} e, \frac{d_{1}}{\left(d_{1} ; e_{1}^{3}\right)}, \frac{d_{2}}{\left(d_{2} ; e_{2}^{4}\right)} ; e_{1}, e_{2}\right) .
$$

Here the addition of $\left(; e_{1}, e_{2}\right)$ to $R^{*}$ denotes that the congruences $C(a, b)$ are replaced by $C\left(e_{1} e_{2}(a, b)\right)$. Since $\left(e_{1} e_{2} ; \Delta\right)=1$ these congruences are

$$
a \equiv \overline{e_{1} e_{2}} a_{0} \quad(\bmod \Delta)
$$

and

$$
b \equiv \overline{e_{1} e_{2}} b_{0} \quad(\bmod \Delta) .
$$

The precise choice of coset has no effect on the above analysis of $R^{*}$ so Lemma 3.2 still holds when different congruence classes are taken for each pair $d_{1}, d_{2}$ in the sum.

Let $\rho\left(d_{1}, d_{2}\right)$ be the number of solutions modulo $d_{1} d_{2}$ to

$$
f(a, b) \equiv 0 \quad\left(\bmod d_{1}\right), \quad b f(a, b) \equiv 0 \quad\left(\bmod d_{2}\right) .
$$


Applying the above analysis to the region $\left(0, d_{1} d_{2}\right]^{2}$ with no congruence $C$ gives the decomposition

$$
\rho\left(d_{1}, d_{2}\right)=\sum_{\substack{e_{1}\left|\psi_{3}\left(d_{1}\right) \\ e_{2}\right| \psi_{4}\left(d_{2}\right)}}\left(\frac{\left(d_{1} ; e_{1}^{3}\right)}{e_{1}} \frac{\left(d_{2} ; e_{2}^{4}\right)}{e_{2}}\right)^{2} \rho^{*}\left(\frac{d_{1}}{\left(d_{1} ; e_{1}^{3}\right)}, \frac{d_{2}}{\left(d_{2} ; e_{2}^{4}\right)}\right) .
$$

It follows that

$$
\begin{aligned}
R & \left.\mathcal{R}, d_{1}, d_{2}\right)-\frac{\rho\left(d_{1}, d_{2}\right) V(\mathcal{R})}{d_{1}^{2} d_{2}^{2} \Delta^{2}} \\
& =\sum_{\substack{e_{1}\left|\psi_{3}\left(d_{1}\right) \\
e_{2}\right| \psi_{4}\left(d_{2}\right)}}\left(R^{*}\left(\mathcal{R} / e_{1} e_{2}, \frac{d_{1}}{\left(d_{1} ; e_{1}^{3}\right)}, \frac{d_{2}}{\left(d_{2} ; e_{2}^{4}\right)}\right)-\frac{V\left(\mathcal{R} / e_{1} e_{2}\right)\left(d_{1} ; e_{1}^{3}\right)^{2}\left(d_{2} ; e_{2}^{4}\right)^{2} \rho^{*}\left(\frac{d_{1}}{\left(d_{1} ; e_{1}^{3}\right)}, \frac{d_{2}}{\left(d_{2} ; e_{2}^{4}\right)}\right)}{d_{1}^{2} d_{2}^{2} \Delta^{2}} .\right.
\end{aligned}
$$

We are interested in the average of this over $d_{1} \leq D_{1}, d_{2} \leq D_{2}$ so we consider

$$
\sum_{\substack{e_{1} f_{1} \leq D_{1}, e_{2} f_{2} \leq D_{2} \\\left(e_{1} f_{1} ; e_{2} f_{2}\right)=\left(e_{1} f_{1} e_{2} f_{2} ; \Delta\right)=1}} \delta\left(e_{1}, e_{2}, f_{1}, f_{2}\right) \max _{P(\mathcal{R}) \leq M}\left|R^{*}\left(\mathcal{R} / e_{1} e_{2}, f_{1}, f_{2}\right)-\frac{\rho^{*}\left(f_{1}, f_{2}\right) V\left(\mathcal{R} / e_{1} e_{2}\right)}{f_{1}^{2} f_{2}^{2}}\right|,
$$

where $\delta\left(e_{1}, e_{2}, f_{1}, f_{2}\right)$ is the number of pairs $d_{1} \leq D_{1}, d_{2} \leq D_{2}$ with

$$
e_{1}\left|\psi\left(d_{1}\right), e_{2}\right| \psi\left(d_{2}\right), f_{1}=\frac{d_{1}}{\left(d_{1} ; e_{1}^{3}\right)}, f_{2}=\frac{d_{2}}{\left(d_{2} ; e_{2}^{4}\right)} .
$$

It is clear that $\delta$ is the product of the number of suitable $d_{1}$ by the number of $d_{2}$. These latter quantities were estimated by Daniel: they are bounded by divisor functions. It follows that for any $\epsilon>0$ we have

$$
\delta\left(e_{1}, e_{2}, f_{1}, f_{2}\right) \ll_{\epsilon}\left(D_{1} D_{2}\right)^{\epsilon} .
$$

Our sum is thus majorised by

$$
\left(D_{1} D_{2}\right)^{\epsilon} \sum_{\substack{e_{1} f_{1} \leq D_{1}, e_{2} f_{2} \leq D_{2} \\\left(e_{1} f_{1} ; e_{2} f_{2}\right)=\left(e_{1} f_{1} e_{2} f_{2} ; \Delta\right)=1}} \max _{P(\mathcal{R}) \leq M}\left|R^{*}\left(\mathcal{R} / e_{1} e_{2}, f_{1}, f_{2}\right)-\frac{\rho^{*}\left(f_{1}, f_{2}\right) V\left(\mathcal{R} / e_{1} e_{2}\right)}{f_{1}^{2} f_{2}^{2}}\right| .
$$

For each pair $\left(e_{1}, e_{2}\right)$ in this sum we apply Lemma 3.2 to the sum over $f_{1}, f_{2}$. This results in a bound

$$
\left(D_{1} D_{2}\right)^{\epsilon} \sum_{e_{1} \leq D_{1}, e_{2} \leq D_{2}}\left(\frac{D_{1} D_{2}}{e_{1} e_{2}}+\frac{M}{e_{1} e_{2}}\left(\left(\frac{D_{1} D_{2}}{e_{1} e_{2}}\right)^{\frac{1}{2}}+\frac{D_{2}}{e_{2}}\right)\right) .
$$

We may therefore conclude with the following level of distribution result.

Lemma 3.3. For any $D_{1}, D_{2}>0$ and any $\epsilon>0$ we have

$$
\begin{aligned}
\sum_{\substack{d_{1} \leq D_{1}, d_{2} \leq D_{2} \\
\left(d_{1} ; d_{2}\right)=\left(d_{1} d_{2} ; \Delta\right)=1}} \max _{P(\mathcal{R}) \leq M}\left|R\left(\mathcal{R}, d_{1}, d_{2}\right)-\frac{\rho\left(d_{1}, d_{2}\right) V(\mathcal{R})}{d_{1}^{2} d_{2}^{2} \Delta^{2}}\right| \\
\ll_{\epsilon}\left(D_{1} D_{2}\right)^{\epsilon}\left(D_{1} D_{2}+M\left(\left(D_{1} D_{2}\right)^{\frac{1}{2}}+D_{2}\right)\right) .
\end{aligned}
$$


We are interested in this result when $\mathcal{R}=(0, N]^{2}$ for large $N$.

Lemma 3.4. Let

$$
R\left(d_{1}, d_{2}\right)=R\left((0, N]^{2}, d_{1}, d_{2}\right) .
$$

Suppose $\eta>0$ is fixed. Then there exists $\delta>0$, depending on $\eta$, such that if

$$
0<D_{1} D_{2} \leq N^{2-\eta}
$$

and

$$
0<D_{2} \leq N^{1-\eta}
$$

we have

$$
\sum_{\substack{d_{1} \leq D_{1}, d_{2} \leq D_{2} \\\left(d_{1} ; d_{2}\right)=\left(d_{1} d_{2} ; \Delta\right)=1}}\left|R\left(d_{1}, d_{2}\right)-\frac{\rho\left(d_{1}, d_{2}\right) N^{2}}{d_{1}^{2} d_{2}^{2} \Delta^{2}}\right| \ll_{\eta} N^{2-\delta}
$$

Proof. This follows on putting $V(\mathcal{R})=N^{2}, P(\mathcal{R}) \ll N$ into the previous lemma and taking $\epsilon$ sufficiently small in terms of $\eta$.

If we let $\rho_{1}(d)$ be the number of solutions modulo $d$ to $f(a, b) \equiv 0(\bmod d)$ and $\rho_{2}(d)$ the number of solutions to $b f(a, b) \equiv 0(\bmod d)$ then if $\left(d_{1} ; d_{2}\right)=1$ we have

$$
\rho\left(d_{1}, d_{2}\right)=\rho_{1}\left(d_{1}\right) \rho_{2}\left(d_{2}\right) .
$$

We also need to understand the quantity

$$
R_{1}\left(d_{1}, d_{2}\right)=\#\left\{(a, b) \in(0, N]^{2}: C(a, b), b f(a, b) \equiv 0\left(\bmod d_{1}\right), b \equiv 0\left(\bmod d_{2}\right)\right\} .
$$

This is only required for small $d_{1}, d_{2}$ so the following is sufficient.

Lemma 3.5. For any $d_{1}, d_{2} \in \mathbb{N}$ with $\left(d_{1} ; d_{2}\right)=\left(d_{1} d_{2} ; \Delta\right)=1$ and $d_{1} d_{2} \leq N$ we have, for any $\epsilon>0$ that

$$
R_{1}\left(d_{1}, d_{2}\right)=\frac{N^{2} \rho_{2}\left(d_{1}\right)}{d_{1}^{2} d_{2} \Delta^{2}}+O_{\epsilon}\left(N d_{1}^{\epsilon}\right)
$$

Proof. The number of points counted by $R_{1}$ congruent to a given solution modulo $d_{1} d_{2} \Delta$ is

$$
\frac{N^{2}}{d_{1}^{2} d_{2}^{2} \Delta^{2}}+O\left(1+\frac{N}{d_{1} d_{2} \Delta}\right)=\frac{N^{2}}{d_{1}^{2} d_{2}^{2} \Delta^{2}}+O\left(\frac{N}{d_{1} d_{2}}\right) .
$$

By the Chinese remainder theorem the number of solutions modulo $d_{1} d_{2} \Delta$ is $d_{2} \rho_{2}\left(d_{1}\right)$. It follows that

$$
R\left(d_{1}, d_{2}\right)=\frac{N^{2} \rho_{2}\left(d_{1}\right)}{d_{1}^{2} d_{2} \Delta^{2}}+O\left(\frac{N \rho_{2}\left(d_{1}\right)}{d_{1}}\right)=\frac{N^{2} \rho_{2}\left(d_{1}\right)}{d_{1}^{2} d_{2} \Delta^{2}}+O_{\epsilon}\left(N d_{1}^{\epsilon}\right) .
$$




\section{The Functions $\rho_{1}$ and $\rho_{2}$}

We need various estimates for sums and products involving the functions $\rho_{1}$ and $\rho_{2}$. Let $\nu(d)$ be the number of solutions to the congruence

$$
f(x) \equiv 0 \quad(\bmod d)
$$

For all primes $p \notin \mathcal{P}_{1}$ we may write $\rho_{1}(p)$ and $\rho_{2}(p)$ in terms of $\nu(p)$ :

$$
\rho_{1}(p)=(p-1) \nu(p)+1
$$

and

$$
\rho_{2}(p)=(p-1) \nu(p)+p .
$$

In the following equations let $c$ denote a real constant which may depend on $q$ and $f$ and which may differ from line to line. It is well known that

$$
\begin{gathered}
\sum_{p \leq x} \frac{1}{p}=\log \log x+c+o(1), \\
\sum_{\substack{p \leq x \\
p \equiv a(\bmod q)}} \frac{1}{p}=\frac{1}{q-1} \log \log x+c+o(1),
\end{gathered}
$$

if $(a ; q)=1$, and

$$
\sum_{p \leq x} \frac{1}{p^{2}}=c+o(1) .
$$

Let $L$ be the cubic field generated by the polynomial $f$ and let $\zeta_{L}$ be its Dedekind zeta function. For all primes $p \notin \mathcal{P}_{1}$ we know that $\nu(p)$ is equal to the coefficient of $p^{-s}$ in $\zeta_{L}(s)$. It follows from the Prime Ideal Theorem that

$$
\sum_{p \leq x} \frac{\nu(p)}{p}=\log \log x+c+o(1) .
$$

Finally we would like to show that, for $(a ; q)=1$, we have

$$
\sum_{\substack{p \leq x \\ p \equiv a}} \frac{\nu(p)}{p} \sim \frac{1}{q-1} \log \log x .
$$

Unfortunately this is not always true. For example, suppose we have

$$
f(t)=t^{3}-7 t^{2}+14 t-7
$$


The field $L$ is then abelian of degree 3 and contained in the cyclotomic field $\mathbb{Q}\left(\zeta_{7}\right)$. It is easy to deduce from this that

$$
\nu(p)= \begin{cases}3 & p \equiv \pm 1 \quad(\bmod 7) \\ 0 & p \equiv \pm 2, \pm 3 \quad(\bmod 7) .\end{cases}
$$

The formula (4) is therefore not true for this $f$ when $q=7$. It follows that many of the details of the sieve would be different in this case. In order to avoid these difficulties we restrict our attention to those polynomials $f$ and primes $q$ for which (4) holds. We will show that (4) follows from our hypothesis that the number field $L$ is not contained in $\mathbb{Q}\left(\zeta_{q}\right)$.

Expanding using characters we are interested in

$$
\frac{1}{q-1} \sum_{\chi} \overline{(\bmod q)} \overline{\chi(a)} \sum_{p \leq x} \frac{\chi(p) \nu(p)}{p} .
$$

For $p \notin \mathcal{P}_{1}$ the quantity $\chi(p) \nu(p)$ is the coefficient of $p^{-s}$ in the function $\zeta_{L}(s, \chi)$. This is the Hecke $L$-function coming from the character which maps an ideal $I$ to $\chi(N(I))$.

Lemma 4.1. If $\chi \neq \chi_{0}$ is a character modulo $q$ then, under our assumption that $L \nsubseteq \mathbb{Q}\left(\zeta_{q}\right)$, $\zeta_{L}(s, \chi)$ is regular at $s=1$.

Proof. We say that a property holds for almost all primes if it holds for all primes with finitely many exceptions. It is enough to show that the Hecke character $I \mapsto \chi(N(I))$ is not induced from the trivial character, as it is the only primitive Hecke character whose $L$-function has a singularity at $s=1$. In other words we need to show that there are infinitely many prime ideals $P$ for which $\chi(N(P)) \neq 1$. We suppose that this is false so that, in particular, almost all primes $p$, for which there is an ideal of norm $p$, are in a proper subgroup $H$ of $(\mathbb{Z} / q \mathbb{Z})^{*}$.

We first consider the case that $L / \mathbb{Q}$ is not Galois, so its discriminant, $\delta$, is not a square. It can be shown that if a prime $p$ satisfies $\left(\frac{\delta}{p}\right)=-1$ then it factorises in $L$ into prime ideals of norms $p$ and $p^{2}$. It follows that the reduction modulo $q$ of almost all such primes must be in $H$. However, we can show using Dirichlet's theorem on primes in arithmetic progressions that for any prime $q$ and any nonsquare integer $\delta$ the reductions modulo $q$ of almost all the primes $p$ for which $\left(\frac{\delta}{p}\right)=-1$ generate the whole of $(\mathbb{Z} / q \mathbb{Z})^{*}$. This is a contradiction so $\chi$ cannot be induced from the trivial character and $\zeta_{L}(s, \chi)$ is regular at 1 .

Next we consider the case that $L / \mathbb{Q}$ is Galois. We know, by assumption, that the primes which split completely in $L$ are contained in $H$.

Suppose in general that we have Galois number fields $L_{1}, L_{2}$ and almost all the primes which split completely in $L_{1}$ also split completely in $L_{2}$. It follows that almost all the primes which split in $L_{1}$ also split in the composite extension $L_{1} L_{2}$. By Chebotarev's Density Theorem the density of primes which split in $L_{1}$ is $\frac{1}{\left[L_{1}: \mathbb{Q}\right]}$ whereas the density of those splitting in $L_{1} L_{2}$ is $\frac{1}{\left[L_{1} L_{2}: \mathbb{Q}\right]}$. We conclude that

$$
\frac{1}{\left[L_{1}: \mathbb{Q}\right]} \leq \frac{1}{\left[L_{1} L_{2}: \mathbb{Q}\right]}
$$


so that $L_{1} L_{2}=L_{1}$ and therefore $L_{2}$ is a subfield of $L_{1}$.

By class field theory we can construct a number field $L_{H}$ whose only ramified prime is $q$ and for which the primes which split completely are those in $H,\left(L_{H}\right.$ is the class field coming from the modulus $(q)$ and the subgroup $H)$. It follows by the previous paragraph that $L_{H}$ is contained in $L$. However, $[L: \mathbb{Q}]=3$ so we must have $L=L_{h}$. By class field theory, $L_{H} \subseteq \mathbb{Q}\left(\zeta_{q}\right)$, which contradicts our assumption on $L$. We deduce that the Hecke character is not induced from the trivial one and thus its $L$-function has no singularities.

It now follows by general theory that for $\chi \neq \chi_{0}$ we have, as $x \rightarrow \infty$,

$$
\sum_{p \leq x} \frac{\chi(p) \nu(p)}{p}=c+o(1)
$$

for some constant $c$ depending on $f, q$ and $\chi$. We can therefore conclude that

$$
\sum_{\substack{p \leq x \\ p \equiv a}} \frac{\nu(p)}{p}=\frac{1}{q-1} \log \log x+c+o(1),
$$

with $c$ depending on $f, q$ and $a$.

Let

$$
\mathcal{P}=\left\{p: p \notin \mathcal{P}_{1}, p \equiv 1 \quad(\bmod q)\right\}
$$

and

$$
\mathcal{P}^{\prime}=\left\{p: p \notin \mathcal{P}_{1}, p \not \equiv 1 \quad(\bmod q)\right\} .
$$

Lemma 4.2. As $x \rightarrow \infty$ we have

$$
\prod_{\substack{p \leq x \\ p \in \mathcal{P}}}\left(1-\frac{\rho_{2}(p)}{p^{2}}\right) \sim \frac{c_{2}(f, q)}{(\log x)^{\frac{2}{q-1}}}
$$

and

$$
\prod_{\substack{p \leq x \\ p \in \mathcal{P}^{\prime}}}\left(1-\frac{\rho_{1}(p)}{p^{2}}\right) \sim \frac{c_{1}(f, q)}{(\log x)^{\frac{q-2}{q-1}}}
$$

where $c_{1}(f, q), c_{2}(f, q)>0$.

Proof. Let

$$
P=\prod_{\substack{p \leq x \\ p \in \mathcal{P}}}\left(1-\frac{\rho_{2}(p)}{p^{2}}\right) .
$$


Since all the primes dividing $f$ are in $\mathcal{P}_{1}$ and hence not in $\mathcal{P}$ we know that $\rho_{2}(p)<p^{2}$ for all $p \in \mathcal{P}$. It follows that the terms in $P$ are all positive so we may take logs:

$$
\begin{aligned}
\log P & =\sum_{\substack{p \leq x \\
p \in \mathcal{P}}} \log \left(1-\frac{\rho_{2}(p)}{p^{2}}\right) \\
& =\sum_{\substack{p \leq x \\
p \in \mathcal{P}}}\left(-\frac{\rho_{2}(p)}{p^{2}}+O\left(\frac{1}{p^{2}}\right)\right) \\
& =\sum_{\substack{p \leq x \\
p \in \mathcal{P}}}\left(-\frac{\nu(p)+1}{p}+O\left(\frac{1}{p^{2}}\right)\right) \\
& =-\frac{2}{q-1} \log \log x+c+o(1) .
\end{aligned}
$$

The first result follows on taking $c_{2}(f, q)=e^{c}$ with the $c$ from the last line. The second result can be proved analogously.

It is clear that if $p \notin \mathcal{P}_{1}$ then $\nu(p) \leq 3$ so that $\rho_{1}(p) \leq 3 p$. We also need a bound for $\rho_{1}$ at prime powers.

Lemma 4.3. For any prime $p \notin \mathcal{P}_{1}$ and any $\alpha \in \mathbb{N}$ we have

$$
\rho_{1}\left(p^{\alpha}\right) \ll p^{\frac{4 \alpha}{3}}
$$

the implied constant being absolute.

Proof. We substitute Daniel's bound [6, (3.2)], which holds for all $p \notin \mathcal{P}_{1}$, into his identity [6. (7.4)]. This results in

$$
\begin{aligned}
\rho_{1}\left(p^{\alpha}\right) & \leq 3 \sum_{0 \leq \beta<\lceil\alpha / 3\rceil} p^{\alpha+\beta}+p^{2(\alpha-\lceil\alpha / 3\rceil)} \\
& \leq 3 \frac{p^{\alpha+\lceil\alpha / 3\rceil}}{p-1}+p^{2(\alpha-\lceil\alpha / 3\rceil)} \ll p^{\frac{4 \alpha}{3}} .
\end{aligned}
$$

As a consequence of this we see that for any $r$ with no prime factors in $\mathcal{P}_{1}$ we have

$$
\rho_{1}(r) \ll r^{\frac{4}{3}} .
$$




\section{The Sum of a Multiplicative Function in an Arith- metic Progression}

Let $g$ be a nonnegative multiplicative function supported on squarefree numbers which satisfies

$$
\sum_{p \leq x} g(p) \log p=k \log x+O(1)
$$

for some $k>0$. If $2 \leq w<z$ we assume that

$$
\prod_{w \leq p<z}(1+g(p)) \ll\left(\frac{\log z}{\log w}\right)^{k} .
$$

We also suppose that

$$
\sum_{p} g(p)^{2} \log p<\infty
$$

Under these assumptions Friedlander and Iwaniec, [8, Theorem A.5], show that

$$
\sum_{m \leq x} g(m)=c_{g}(\log x)^{k}+O\left((\log x)^{k-1}\right),
$$

where

$$
c_{g}=\frac{1}{\Gamma(k+1)} \prod_{p}\left(1-\frac{1}{p}\right)^{k}(1+g(p)) .
$$

We require the following modified version of this result.

Lemma 5.1. Let $g$ be a nonnegative multiplicative function supported on squarefree numbers which satisfies (5), (6) and (7) for some $k>0$. Let $q>2$ be prime. Suppose that $g(q)=0$ and that for all primes $p \equiv 1(\bmod q)$ we have $g(p)=0$. Finally suppose that if $a \not \equiv 0,1$ $(\bmod q)$ then

$$
\sum_{\substack{p \leq x \\ p \equiv a}} g(p) \log p=\frac{1}{q-2} k \log x+O(1) .
$$

Then, for any a with $(a ; q)=1$ we have

$$
\sum_{\substack{m \leq x \\ m \equiv a}} g(m)=\left(\frac{c_{g}}{q-1}+o(1)\right)(\log x)^{k} .
$$

Proof. Let

$$
M_{g}(x)=\sum_{\substack{m \leq x \\ m \equiv a}} g(m)
$$


We begin by considering

$$
\sum_{\substack{m \leq x \\ m \equiv a}} g(m) \log m=\sum_{\substack{n p \leq x \\ n p \equiv a}} g(n p) \log p .
$$

Using that $g$ is multiplicative and supported on squarefree numbers coprime to $q$ this can be written as

$$
\sum_{n \leq x} g(n) \sum_{\substack{p \leq x / n \\ p \equiv a \bar{n}}} g(p) \log p-\sum_{\substack{n p^{2} \leq x \\ n p^{2} \equiv a}} g(n p) g(p) \log p .
$$

From (6) we get

$$
\sum_{n \leq x} g(n) \leq \prod_{p \leq x}(1+g(p)) \ll(\log x)^{k}
$$

and from (7) we deduce

$$
\sum_{n p^{2} \leq x} g(n p) g(p) \log p \ll \sum_{n \leq x} g(n) \sum_{p} g(p)^{2} \log p \ll(\log x)^{k} .
$$

Using these bounds as well as (9) the above sum becomes

$$
\frac{k}{q-2} \sum_{\substack{n \leq x \\ n \neq a}} g(n)(\log x-\log n)+O\left((\log x)^{k}\right) .
$$

We can write this as

$$
\frac{k}{q-2}\left(\sum_{n \leq x} g(n)(\log x-\log n)-\sum_{\substack{n \leq x \\ n \equiv a(\bmod q)}} g(n)(\log x-\log n)\right)+O\left((\log x)^{k}\right) .
$$

From the bound (8) we have

$$
\sum_{n \leq x} g(n)=c_{g}(\log x)^{k}+O\left((\log x)^{k-1}\right)
$$

and, summing by parts,

$$
\left.\sum_{n \leq x} g(n) \log n=\frac{k c_{g}}{k+1}(\log x)^{k+1}+O\left((\log x)^{k}\right)\right) .
$$

Our sum is thus

$$
\frac{k}{q-2}\left(\frac{c_{g}}{k+1}(\log x)^{k+1}-\sum_{\substack{n \leq x \\ n \equiv a}} g(n)(\log x-\log n)\right)+O\left((\log x)^{k}\right) .
$$


We therefore get

$$
(q-2-k) \sum_{\substack{m \leq x \\ m \equiv a}} g(m) \log m+k \log x M_{g}(x)-\frac{k c_{g}}{k+1}(\log x)^{k+1} \ll(\log x)^{k} .
$$

Since

$$
\log x M_{g}(x)-\sum_{\substack{m \leq x \\ m \equiv a}} g(m) \log m=\sum_{\substack{m \leq x \\ m \equiv a}} g(m) \log \frac{x}{m}=\int_{1}^{x} M_{g}(t) t^{-1} d t
$$

we have

$$
M_{g}(x) \log x-\left(1-\frac{k}{q-2}\right) \int_{1}^{x} M_{g}(t) t^{-1} d t-\frac{k c_{g}}{(k+1)(q-2)}(\log x)^{k+1} \ll(\log x)^{k} .
$$

We therefore conclude that for $x \geq 2$

$$
M_{g}(x) \log x-\left(1-\frac{k}{q-2}\right) \int_{2}^{x} M_{g}(t) t^{-1} d t-\frac{k c_{g}}{(k+1)(q-2)}(\log x)^{k+1} \ll(\log x)^{k} .
$$

Let

$$
l=1-\frac{k}{q-2}
$$

so that this is

$$
M_{g}(x) \log x-l \int_{2}^{x} M_{g}(t) t^{-1} d t-\frac{k c_{g}}{(k+1)(q-2)}(\log x)^{k+1} \ll(\log x)^{k} .
$$

Dividing by $x(\log x)^{l+1}$ we then get

$$
\begin{gathered}
x^{-1}(\log x)^{-l}\left(M_{g}(x)-l(\log x)^{-1} \int_{2}^{x} M_{g}(t) t^{-1} d t-\frac{k c_{g}}{(k+1)(q-2)}(\log x)^{k}\right) \\
\ll x^{-1}(\log x)^{k-l-1} .
\end{gathered}
$$

We integrate this from 2 to $x$, replacing $x$ by $t$ and $t$ by $u$. For any $\epsilon>0$ the RHS will be

$$
\int_{2}^{x} t^{-1}(\log t)^{k-l-1} d t \ll_{\epsilon} 1+(\log x)^{k-l+\epsilon},
$$

and the LHS will be

$$
\int_{2}^{x} t^{-1}(\log t)^{-l}\left(M_{g}(t)-l(\log t)^{-1} \int_{2}^{t} M_{g}(u) u^{-1} d u-\frac{k c_{g}}{(k+1)(q-2)}(\log t)^{k}\right) d t .
$$

Re-ordering the double integral we see that

$$
(\log x)^{-l} \int_{2}^{x} M_{g}(t) t^{-1} d t-\frac{k c_{g}}{(k+1)(q-2)} \int_{2}^{x} t^{-1}(\log t)^{k-l} d t \ll 1+(\log x)^{k-l+\epsilon} .
$$


However

$$
M_{g}(x) \log x-\frac{k c_{g}}{(k+1)(q-2)}(\log x)^{k+1}+O\left((\log x)^{k}\right)=l \int_{2}^{x} M_{g}(t) t^{-1} d t
$$

so this simplifies to

$$
\begin{gathered}
M_{g}(x)=\frac{k c_{g}}{(k+1)(q-2)}\left((\log x)^{k}+l(\log x)^{l-1} \int_{2}^{x} t^{-1}(\log t)^{k-l} d t\right) \\
+O\left((\log x)^{l-1}+(\log x)^{k-1+\epsilon}\right) .
\end{gathered}
$$

We know that

$$
k-l=k-1+\frac{k}{q-2}>-1
$$

So

$$
\begin{aligned}
M_{g}(x) & =k c_{g} \frac{1+l(k-l+1)^{-1}}{(k+1)(q-2)}(\log x)^{k}+o\left((\log x)^{k}\right) \\
& =k c_{g} \frac{(k q-2 k+q-2) /(k q-k)}{(k+1)(q-2)}(\log x)^{k}+o\left((\log x)^{k}\right) \\
& =\frac{c_{g}}{q-1}(\log x)^{k}+o\left((\log x)^{k}\right) .
\end{aligned}
$$

\section{The Sieve}

\subsection{The Sieve Decomposition}

Let $\mathcal{A}=\left(a_{n}\right)$ be the sequence given by

$$
a_{n}=\sum_{\substack{(a, b) \in(0, N]^{2} \\ C(a, b), b f(a, b)=n}} 1
$$

We will sieve $\mathcal{A}$ by the set of primes

$$
\mathcal{P}=\left\{p: p \notin \mathcal{P}_{1}, p \equiv 1 \quad(\bmod q)\right\} .
$$

Let

$$
x=\max \left\{f(a, b):(a, b) \in(0, N]^{2}\right\}=(c+o(1)) N^{3},
$$

for some constant $c$ which depends on $f$. We wish to prove a positive lower bound for the sifting function

$$
S(\mathcal{A}, \mathcal{P}, x)=\sum_{(n ; P(x))=1} a_{n}
$$


where

$$
P(z)=\prod_{\substack{p<z \\ p \in \mathcal{P}}} p
$$

Applying the Buchstab identity we get, for some $\alpha \in\left(\frac{1}{2}, 1\right)$, that

$$
S(\mathcal{A}, \mathcal{P}, x)=S\left(\mathcal{A}, \mathcal{P}, N^{\alpha}\right)-\sum_{\substack{N^{\alpha} \leq p<x \\ p \in \mathcal{P}}} S\left(\mathcal{A}_{p}, \mathcal{P}, p\right)
$$

If a prime $p$ divides $b f(a, b)$ then either $p \mid b$ or $p \mid f(a, b)$. We may therefore write

$$
S(\mathcal{A}, \mathcal{P}, x) \geq S\left(\mathcal{A}, \mathcal{P}, N^{\alpha}\right)-\sum_{\substack{N^{\alpha} \leq p<x \\ p \in \mathcal{P}}}\left(S\left(\mathcal{A}_{p}^{(1)}, \mathcal{P}, p\right)+S\left(\mathcal{A}_{p}^{(2)}, \mathcal{P}, p\right)\right)
$$

where $\mathcal{A}_{p}^{(1)}$ is the subsequence of $\mathcal{A}_{p}$ coming from pairs $(a, b)$ with $p \mid b$ whereas $\mathcal{A}_{p}^{(2)}$ is the subsequence coming from $p \mid f(a, b)$.

If $p \mid b$ we must have $p \leq N$ so we can truncate the sum over $\mathcal{A}_{p}^{(1)}$ to $p \leq N$. As our level of distribution, Lemma 3.4, is only nontrivial for $D_{1} D_{2} \leq N^{2}$ we split the sum over $\mathcal{A}_{p}^{(2)}$ at $N^{\beta}$ for some $\beta \in\left(\frac{3}{2}, 2\right)$. We conclude that

$$
S(\mathcal{A}, \mathcal{P}, x) \geq S_{1}-S_{2}-S_{3}-S_{4}
$$

where

$$
\begin{gathered}
S_{1}=S\left(\mathcal{A}, \mathcal{P}, N^{\alpha}\right), \\
S_{2}=\sum_{\substack{N^{\alpha} \leq p \leq N \\
p \in \mathcal{P}}} S\left(\mathcal{A}_{p}^{(1)}, \mathcal{P}, p\right), \\
S_{3}=\sum_{\substack{N^{\alpha} \leq p<N^{\beta} \\
p \in \mathcal{P}}} S\left(\mathcal{A}_{p}^{(2)}, \mathcal{P}, p\right)
\end{gathered}
$$

and

$$
S_{4}=\sum_{\substack{N^{\beta} \leq p<x \\ p \in \mathcal{P}}} S\left(\mathcal{A}_{p}^{(2)}, \mathcal{P}, p\right)
$$

We then need a lower bound for $S_{1}$ and upper bounds for $S_{2}, S_{3}$ and $S_{4}$. All our bounds will eventually depend on the $\beta$-sieve as given by Friedlander and Iwaniec in [ㅇ, Theorem 11.13]. We let $A_{1}, B_{1}$ denote the constants $A, B$ in the sieve of dimension $\frac{2}{q-1}$ and $A_{2}, B_{2}$ those for the sieve of dimension $\frac{q-2}{q-1}$. These are the only sieves we will use.

Throughout this section $q, f$ and $\Delta$ are fixed. All use of the notation $o$ is as $N \rightarrow \infty$. 


\subsection{The Sum $S_{1}$}

We have

$$
S_{1}=S\left(\mathcal{A}, \mathcal{P}, N^{\alpha}\right)
$$

Since $\alpha<1$ we can take $D_{1}=1$ and $D_{2}=N^{\alpha}$ in Lemma 3.4. This shows that we can apply a lower bound sieve of level $N^{\alpha}$ as the remainder term is $O\left(N^{2-\delta}\right)$ for some $\delta>0$. Using the notation of [8] we have

$$
X=\frac{N^{2}}{\Delta^{2}}
$$

and $g(p)$ is the multiplicative function given by

$$
g(p)= \begin{cases}\frac{\rho_{2}(p)}{p^{2}} & p \in \mathcal{P} \\ 0 & \text { Otherwise }\end{cases}
$$

It follows from Lemma 4.2 that the sieve dimension is $\frac{2}{q-1}$. If $q>5$ then

$$
\frac{2}{q-1}<\frac{1}{2}
$$

and so the sifting limit is 1 . We may therefore use the lower bound sieve to deduce that

$$
S_{1} \geq \frac{\left(B_{1}+o(1)\right) N^{2}}{\Delta^{2}} \prod_{\substack{p<N^{\alpha} \\ p \in \mathcal{P}}}\left(1-\frac{\rho_{2}(p)}{p^{2}}\right)+O\left(N^{2-\delta}\right) .
$$

Applying Lemma 4.2 we conclude that

$$
S_{1} \geq \frac{\left(c_{2}(f, q) B_{1}+o(1)\right) N^{2}}{\Delta^{2}(\alpha \log N)^{\frac{2}{q-1}}} .
$$

Finally, for any $\epsilon>0$ we can choose $\alpha$ sufficiently close to 1 in terms of $\epsilon$ to get the bound

$$
S_{1} \geq \frac{\left(c_{2}(f, q) B_{1}-\epsilon+o(1) N^{2}\right.}{\Delta^{2}(\log N)^{\frac{2}{q-1}}} .
$$

\subsection{The Sum $S_{2}$}

In our bound for $S_{2}$ we will exploit the fact that $\alpha$ may be taken as close to 1 as we require. We therefore do not need to give a bound which is as sharp as possible. It is enough to show that for any $\epsilon>0$ we can choose $\alpha<1$ depending on $\epsilon$ such that

$$
S_{2} \leq \frac{(\epsilon+o(1)) N^{2}}{(\log N)^{\frac{2}{q-1}}} .
$$


We have

$$
S_{2} \leq \sum_{\substack{N^{\alpha} \leq p \leq N \\ p \in \mathcal{P}}} S\left(\mathcal{A}_{p}^{(1)}, \mathcal{P}, N^{\alpha}\right) .
$$

For each pair $(a, b)$ counted by $S_{2}$ we may write $b=p r$ where

$$
\begin{gathered}
p \in \mathcal{P} \cap\left[N^{\alpha}, N\right], \\
r \leq N^{1-\alpha}=R
\end{gathered}
$$

and

$$
(r ; P(R))=1 .
$$

In addition we have $b \equiv b_{0}(\bmod \Delta)$. By our construction of $b_{0}$ we know that for each $p^{\prime} \mid \Delta$ there exists an $l$ for which $p^{\prime l} \mid \Delta$ and

$$
b_{0} \not \equiv 0 \quad\left(\bmod p^{\prime l}\right) .
$$

It follows that for each such prime its power dividing $b$ is the same as that dividing $b_{0}$. For $p \in \mathcal{P}$ we have $(\Delta ; p)=1$. It follows that for each $p^{\prime} \mid \Delta$ the power of $p^{\prime}$ dividing $r$ is precisely that dividing $b_{0}$. In other words we may write

$$
r=\left(b_{0} ; \Delta\right) r^{\prime} \text { with }\left(r^{\prime} ; \Delta\right)=1 .
$$

Given such an $r$ and a pair $(a, b)$ satisfying $C(a, b)$ the condition $r \mid b$ is equivalent to $r^{\prime} \mid b$.

We may therefore write

$$
S_{2} \leq \sum_{\substack{r \leq R /\left(b_{0} ; \Delta\right) \\(r ; P(R) \Delta)=1}} S_{2}(r)
$$

where

$$
S_{2}(r)=\#\left\{(a, b) \in(0, N]^{2}: C(a, b), r \mid b, b / r\left(b_{0} ; \Delta\right) \in \mathcal{P},\left(b f(a, b) ; P\left(N^{\alpha}\right)\right)=1\right\} .
$$

Note that the variable of summation, $r$, is $r^{\prime}$ in the above notation.

Recall that

$$
\mathcal{P}^{\prime}=\left\{p: p \notin \mathcal{P}_{1}, p \not \equiv 1 \quad(\bmod q)\right\}
$$

and let

$$
P^{\prime}(z)=\prod_{\substack{p<z \\ p \in \mathcal{P}^{\prime}}} p
$$

If we let $z=N^{\delta}$ for some $\delta>0$ then provided $\delta<\alpha$ we have

$$
S_{2}(r) \leq \#\left\{(a, b) \in(0, N]^{2}: C(a, b), r \mid b,(b f(a, b) ; P(z))=\left(b / r ; P^{\prime}(z)\right)=1\right\} .
$$


Suppose that $\mu_{1}^{+}, \mu_{2}^{+}$are upper bound sieves of level $z$. We have

$$
S_{2}(r) \leq \sum_{\substack{(a, b) \in(0, N]^{2} \\ r \mid b, C(a, b)}}\left(\sum_{d \mid(P(z) ; b f(a, b))} \mu_{1}^{+}(d)\right)\left(\sum_{e \mid\left(b / r ; P^{\prime}(z)\right)} \mu_{2}^{+}(e)\right) .
$$

Reordering the summations we get

$$
\begin{aligned}
S_{2}(r) & \leq \sum_{d \mid P(z)} \sum_{e \mid P^{\prime}(z)} \mu_{1}^{+}(d) \mu_{2}^{+}(e) \#\left\{(a, b) \in(0, N]^{2}: C(a, b), r e|b, d| b f(a, b)\right\} \\
& =\sum_{d \mid P(z)} \sum_{e \mid P^{\prime}(z)} \mu_{1}^{+}(d) \mu_{2}^{+}(e) R_{1}(d, r e) .
\end{aligned}
$$

If $\delta$ is sufficiently small so that

$$
1-\alpha+2 \delta<1
$$

then

$$
r d e \leq N
$$

Furthermore $(r e ; d)=(d r e ; \Delta)=1$ so Lemma 3.5 applies and we get

$$
S_{2}(r) \leq \sum_{d \mid P(z)} \sum_{e \mid P^{\prime}(z)} \mu_{1}^{+}(d) \mu_{2}^{+}(e)\left(\frac{N^{2} \rho_{2}(d)}{d^{2} r e \Delta^{2}}+O_{\epsilon}\left(N d^{\epsilon}\right)\right) .
$$

The contribution of the error term to $S_{2}$ is bounded by

$$
\sum_{r \leq R} N^{1+2 \delta+\epsilon} \ll N^{2-\alpha+2 \delta+\epsilon}=o\left(\frac{N^{2}}{(\log N)^{\frac{2}{q-1}}}\right),
$$

in view of our assumption on the size of $\delta$.

The main term in the above estimate for $S_{2}(r)$ is

$$
\frac{N^{2}}{r \Delta^{2}}\left(\sum_{d \mid P(z)} \mu_{1}^{+}(d) \frac{\rho_{2}(d)}{d^{2}}\right)\left(\sum_{e \mid P^{\prime}(z)} \frac{\mu_{2}^{+}(e)}{e}\right) .
$$

The two sums may now be estimated using the sieve. We let $\left(\mu_{1}^{+}\right)$be a sieve of dimension $\frac{2}{q-1}$. It follows using Lemma 4.2 that

$$
\begin{aligned}
\sum_{d \mid P(z)} \mu_{1}^{+}(d) \frac{\rho_{2}(d)}{d^{2}} & \leq\left(A_{1}+o(1)\right) \prod_{\substack{p<z \\
p \in \mathcal{P}}}\left(1-\frac{\rho_{2}(p)}{p^{2}}\right) \\
& =\left(A_{1}+o(1)\right) \frac{c_{2}(f, q)}{(\delta \log N)^{\frac{2}{q-1}}}
\end{aligned}
$$


We let $\left(\mu_{2}^{+}\right)$be a sieve of dimension $\frac{q-2}{q-1}$ and thus we get

$$
\sum_{e \mid P^{\prime}(z)} \frac{\mu_{2}^{+}(e)}{e} \leq\left(A_{2}+o(1)\right) \prod_{\substack{p<z \\ p \in \mathcal{P}^{\prime}}}\left(1-\frac{1}{p}\right)
$$

Finally we have the bound

$$
\sum_{\substack{r \leq R /\left(b_{0} ; \Delta\right) \\(r ; P(R) \Delta=1}} \frac{1}{r} \leq \prod_{\substack{p<R \\ p \in \mathcal{P}^{\prime}}}\left(1-\frac{1}{p}\right)^{-1}
$$

By taking $\alpha$ sufficiently close to 1 we can assume that $R<z$. It follows that

$$
\prod_{\substack{p<z \\ p \in \mathcal{P}^{\prime}}}\left(1-\frac{1}{p}\right) \prod_{\substack{p<R \\ p \in \mathcal{P}^{\prime}}}\left(1-\frac{1}{p}\right)^{-1}=\prod_{\substack{R<p<z \\ p \in \mathcal{P}^{\prime}}}\left(1-\frac{1}{p}\right) \sim\left(\frac{1-\alpha}{\delta}\right)^{\frac{q-2}{q-1}} .
$$

We finally conclude that

$$
S_{2} \leq\left(\frac{1-\alpha}{\delta}\right)^{\frac{q-2}{q-1}} \frac{\left(A_{1} A_{2} c_{2}(f, q)+o(1)\right) N^{2}}{\Delta^{2}(\delta \log N)^{\frac{2}{q-1}}} .
$$

It follows that for any $\epsilon>0$ there exists an $\alpha<1$ depending on $\epsilon$ such that

$$
S_{2} \leq \frac{(\epsilon+o(1)) N^{2}}{(\log N)^{\frac{2}{q-1}}} .
$$

\subsection{The Sum $S_{3}$}

Let $S_{3}\left(P_{1}, P_{2}\right)$ denote the part of $S_{3}$ with $P_{1} \leq p<P_{2}$. We have

$$
S_{3}\left(P_{1}, P_{2}\right) \leq \sum_{\substack{P_{1} \leq p<P_{2} \\ p \in \mathcal{P}}} S\left(\mathcal{A}_{p}^{(2)}, \mathcal{P}, P_{1}\right)
$$

We will apply an upper bound sieve to each summand separately. For each prime $p$ and each $d \in \mathbb{N}$ we have

$$
\sum_{n \equiv 0}\left(a_{p}^{(2)}\right)_{n}=R(p, d)
$$

If $d<p$ then clearly $(d ; p)=1$. We will apply Lemma 3.4 with $D_{1}=P_{2}$ and

$$
D_{2}=D_{2}\left(P_{1}, P_{2}\right)=\min \left(N^{1-\gamma}, P_{1}, \frac{N^{2-\gamma}}{P_{2}}\right)
$$

for some $\gamma>0$ which we will choose arbitrarily small. We then have

$$
\sum_{\substack{p \leq P_{2}, d \leq D_{2} \\(d ; \Delta)=1, \mu(d)^{2}=1}}\left|R(p, d)-\frac{\rho_{1}(p) \rho_{2}(d) N^{2}}{p^{2} d^{2} \Delta^{2}}\right| \ll N^{2-\delta}
$$


for some $\delta>0$ which depends on $\gamma$.

Applying the upper bound sieve of dimension $\frac{2}{q-1}$ results in

$$
S_{3}\left(P_{1}, P_{2}\right) \leq \frac{\left(A_{1}+o(1)\right) N^{2}}{\Delta^{2}}\left(\prod_{\substack{p<D_{2} \\ p \in \mathcal{P}}}\left(1-\frac{\rho_{2}(p)}{p^{2}}\right)\right) \sum_{\substack{1 \leq p<P_{2} \\ p \in \mathcal{P}}} \frac{\rho_{1}(p)}{p^{2}}+O\left(N^{2-\delta}\right) .
$$

We can evaluate the product using Lemma 4.2 to get

$$
S_{3}\left(P_{1}, P_{2}\right) \leq \frac{\left(c_{2}(f, q) A_{1}+o(1)\right) N^{2}}{\Delta^{2}\left(\log D_{2}\right)^{\frac{2}{q-1}}} \sum_{\substack{P_{1} \leq p<P_{2} \\ p \in \mathcal{P}}} \frac{\rho_{1}(p)}{p^{2}}+O\left(N^{2-\delta}\right) .
$$

In addition, using our previous convention that the value $c$ may vary from line to line, we have

$$
\begin{aligned}
\sum_{\substack{p \leq x \\
p \in \mathcal{P}}} \frac{\rho_{1}(p)}{p^{2}} & =\sum_{\substack{p \leq x \\
p \in \mathcal{P}}} \frac{(p-1) \nu(p)+1}{p^{2}} \\
& =\sum_{\substack{p \leq x \\
p \in \mathcal{P}}} \frac{\nu(p)}{p}+c+o(1) \\
& =\frac{1}{q-1} \log \log x+c+o(1) .
\end{aligned}
$$

We first consider $S_{3}\left(N^{\alpha}, N^{2-\alpha}\right)$. As in the previous section we will take $\alpha$ close to 1 which is enough to make this part of the sum small. We take $D_{2}=N^{\alpha}$, getting

$$
S_{3}\left(N^{\alpha}, N^{2-\alpha}\right) \leq \frac{1}{q-1}(\log (2-\alpha)-\log \alpha) \frac{\left(c_{2}(f, q) A_{1}+o(1)\right) N^{2}}{\Delta^{2}(\alpha \log N)^{\frac{2}{q-1}}} .
$$

It follows that for any $\epsilon>0$ we can choose $\alpha$ sufficiently close to 1 to deduce that

$$
S_{3}\left(N^{\alpha}, N^{2-\alpha}\right) \leq \frac{(\epsilon+o(1)) N^{2}}{(\log N)^{\frac{2}{q-1}}} .
$$

It remains to estimate $S_{3}\left(N^{2-\alpha}, N^{\beta}\right)$. We divide this range into dyadic intervals $\left[P_{1}, 2 P_{1}\right)$. For each such interval we have

$$
D_{2}=\frac{N^{2-\gamma}}{P_{1}} .
$$

By taking $\gamma<2-\beta$ we have $D_{2} \geq 1$ for all the dyadic intervals. In addition if $p \sim P_{1}$ then

$$
\left(\log D_{2}\right)^{-\frac{2}{q-1}}=\left(\log \frac{N^{2-\gamma}}{P_{1}}\right)^{-\frac{2}{q-1}} \leq\left(\log \frac{N^{2-\gamma}}{p}\right)^{-\frac{2}{q-1}} .
$$


We therefore have

$$
S_{3}\left(P_{1}, 2 P_{1}\right) \leq \frac{\left(c_{2}(f, q) A_{1}+o(1)\right) N^{2}}{\Delta^{2}} \sum_{\substack{P_{1} \leq p<2 P_{1} \\ p \in \mathcal{P}}} \frac{\rho_{1}(p)}{p^{2}\left(\log \frac{N^{2-\gamma}}{p}\right)^{\frac{2}{q-1}}}+O\left(N^{2-\delta}\right)
$$

and thus

$$
S_{3}\left(N^{2-\alpha}, N^{\beta}\right) \leq \frac{\left(c_{2}(f, q) A_{1}+o(1)\right) N^{2}}{\Delta^{2}(\log N)^{\frac{2}{q-1}}} \sum_{\substack{N^{2-\alpha} \leq p<N^{\beta} \\ p \in \mathcal{P}}} \frac{\rho_{1}(p)}{p^{2}(2-\gamma-\log p / \log N)^{\frac{2}{q-1}}} .
$$

We have

$$
\sum_{\substack{N \leq p<t \\ p \in \mathcal{P}}} \frac{\rho_{1}(p)}{p^{2}}=\frac{1}{q-1}(\log \log t-\log \log N)+o(1),
$$

so we can sum by parts to get

$$
\begin{aligned}
& \sum_{\substack{N^{2-\alpha} \leq p<N^{\beta} \\
p \in \mathcal{P}}} \frac{\rho_{1}(p)}{p^{2}(2-\gamma-\log p / \log N)^{\frac{2}{q-1}}} \\
\leq & \sum_{\substack{N \leq p<N^{\beta} \\
p \in \mathcal{P}}} \frac{\rho_{1}(p)}{p^{2}(2-\gamma-\log p / \log N)^{\frac{2}{q-1}}} \\
= & \frac{1}{q-1} \frac{\log \beta}{(2-\gamma-\beta)^{\frac{2}{q-1}}}-\frac{2}{(q-1)^{2}} \int_{N}^{N^{\beta}} \frac{\log \log t-\log \log N}{t \log N(2-\gamma-\log t / \log N))^{\frac{q+1}{q-1}}} d t+o(1) \\
= & \frac{1}{q-1} \frac{\log \beta}{(2-\gamma-\beta)^{\frac{2}{q-1}}}-\frac{2}{(q-1)^{2}} \int_{1}^{\beta} \log s(2-\gamma-s)^{-\frac{q+1}{q-1}} d s+o(1) \\
= & \frac{1}{q-1} \int_{1}^{\beta}(2-\gamma-s)^{-\frac{2}{q-1}} \frac{d s}{s}+o(1) .
\end{aligned}
$$

We conclude that

$$
S_{3}\left(N^{2-\alpha}, N^{\beta}\right) \leq \frac{\left(c_{2}(f, q) A_{1}+o(1)\right) N^{2}}{\Delta^{2}(q-1)(\log N)^{\frac{2}{q-1}}} \int_{1}^{\beta}(2-\gamma-s)^{-\frac{2}{q-1}} \frac{d s}{s} .
$$

Combining the above bounds we see that for any $\epsilon>0$ we can choose $\alpha$ sufficiently close to 1 and $\gamma$ sufficiently small to get the bound

$$
S_{3} \leq \frac{\left(c_{2}(f, q) A_{1}+\epsilon+o(1)\right) N^{2}}{\Delta^{2}(q-1)(\log N)^{\frac{2}{q-1}}} \int_{1}^{\beta}(2-s)^{-\frac{2}{q-1}} \frac{d s}{s} .
$$




\subsection{The Sum $S_{4}$}

We have

$$
S_{4} \leq \sum_{\substack{N^{\beta} \leq p<x \\ p \in \mathcal{P}}} S\left(\mathcal{A}_{p}^{(2)}, \mathcal{P}, N^{\beta}\right) .
$$

For each pair $(a, b)$ counted by $S_{4}$ we can write $f(a, b)=p r$ where

$$
\begin{gathered}
p \in\left[N^{\beta}, x\right] \cap \mathcal{P}, \\
r \leq \frac{x}{N^{\beta}}=R
\end{gathered}
$$

and

$$
(r ; P(R))=1 .
$$

Let $f_{0}=f\left(a_{0}, b_{0}\right)$. For each prime $p^{\prime} \mid \Delta$ we know that there exists an $l$ for which $p^{\prime l} \mid \Delta$ and

$$
f_{0} \not \equiv 0 \quad\left(\bmod p^{l}\right) \text {. }
$$

It follows that the power of $p^{\prime}$ dividing $f(a, b)$ is the same as that dividing $f_{0}$. Since $(p ; \Delta)=1$ this power is the same as that dividing $r$. In other words we can write

$$
r=\left(f_{0} ; \Delta\right) r^{\prime}, \quad\left(r^{\prime} ; \Delta\right)=1 .
$$

Given a pair $(a, b)$ with $C(a, b)$ the condition $r \mid f(a, b)$ is equivalent to $r^{\prime} \mid f(a, b)$.

The prime $q$ divides $\Delta$. In addition since $p \in \mathcal{P}$ we have $p \equiv 1(\bmod q)$. It follows that there exists $r_{0}$ depending only on $a_{0}, b_{0}, \Delta$ with $\left(r_{0} ; q\right)=1$ such that

$$
r^{\prime} \equiv r_{0} \quad(\bmod q)
$$

We may now write

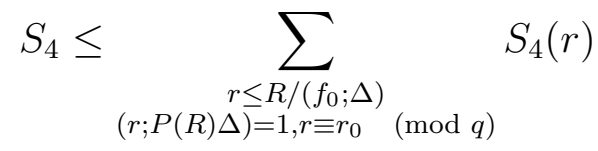

where

$$
S_{4}(r)=\#\left\{(a, b) \in(0, N]^{2}: C(a, b), r \mid f(a, b),\left(f(a, b) / r ; P^{\prime}\left(z^{\prime}\right)\right)=(b f(a, b) ; P(z))=1\right\},
$$

for some $z, z^{\prime}$ satisfying $0 \leq z, z^{\prime} \leq N^{\beta}$. Note that the variable of summation, $r$, is $r^{\prime}$ in the above notation. We will split the sum over $r$ into dyadic segments $R_{1} \leq r<2 R_{1}$.

Let $\mu_{1}^{+}, \mu_{2}^{+}$be upper bound sieves of levels $D^{\prime}$ and $D$, respectively, where $D, D^{\prime}$ depend on $R_{1}$. It follows that

$$
\begin{aligned}
S_{4}(r) & \leq \sum_{\substack{d \mid P^{\prime}\left(z^{\prime}\right) \\
(d ; r)=1}} \sum_{e \mid P(z)} \mu_{1}^{+}(d) \mu_{2}^{+}(e) \#\left\{(a, b) \in(0, N]^{2}: C(a, b), d r|f(a, b), e| b f(a, b)\right\} \\
& =\sum_{\substack{d \mid P^{\prime}\left(z^{\prime}\right) \\
(d ; r)=1}} \sum_{e \mid P(z)} \mu_{1}^{+}(d) \mu_{2}^{+}(e) R(d r, e) .
\end{aligned}
$$


Since $(d r ; e)=(d r e ; \Delta)=1$ we may apply Lemma 3.4. If we write $D=N^{\eta}, D^{\prime}=N^{\eta^{\prime}}$ this requires that

$$
\eta \leq 1-\delta
$$

and

$$
\eta+\eta^{\prime}+\frac{\log R_{1}}{\log N} \leq 2-\delta,
$$

for some $\delta>0$ which we will eventually take arbitrarily small.

Given these assumptions on $\eta$ and $\eta^{\prime}$ the contribution of the error term to $S_{4}$ is $o\left(\frac{N^{2}}{\log N^{\frac{2}{q-1}}}\right)$.

It remains to deal with the main term coming from Lemma 3.4. This is

$$
\frac{N^{2} \rho_{1}(r)}{r^{2} \Delta^{2}}\left(\sum_{\substack{d \mid P^{\prime}\left(z^{\prime}\right) \\(d ; r)=1}} \mu_{1}^{+}(d) \frac{\rho_{1}(d)}{d^{2}}\right)\left(\sum_{e \mid P(z)} \mu_{2}^{+}(e) \frac{\rho_{2}(e)}{e^{2}}\right) .
$$

The two terms can now be estimated using the sieve. Considering the results of Lemma 4.2 we let $\mu_{1}^{+}$be a sieve of dimension $\frac{q-2}{q-1}$ and we let $\mu_{2}^{+}$be a sieve of dimension $\frac{2}{q-1}$. We may assume that $z=D$ and either $z^{\prime}=D^{\prime}$ or $z^{\prime}=N^{\beta} \leq D^{\prime} \leq N^{2}$. It follows that the values of $z, z^{\prime}$ do not affect the sieve upper bounds and therefore

$$
\begin{aligned}
\sum_{\substack{d \mid P^{\prime}\left(z^{\prime}\right) \\
(d ; r)=1}} \mu_{1}^{+}(d) \frac{\rho_{1}(d)}{d^{2}} & \leq\left(A_{2}+o(1)\right) \prod_{\substack{p<D^{\prime} \\
p \in \mathcal{P}^{\prime}}}\left(1-\frac{\rho_{1}(p)}{p^{2}}\right) \prod_{p \mid r}\left(1-\frac{\rho_{1}(p)}{p^{2}}\right)^{-1} \\
& =\frac{\left(c_{1}(f, q) A_{2}+o(1)\right)}{\left(\eta^{\prime} \log N\right)^{\frac{q-2}{q-1}}} \prod_{p \mid r}\left(1-\frac{\rho_{1}(p)}{p^{2}}\right)^{-1}
\end{aligned}
$$

and

$$
\sum_{e \mid P(z)} \mu_{2}^{+}(e) \frac{\rho_{2}(e)}{e^{2}} \leq\left(A_{1}+o(1)\right) \prod_{\substack{p<z \\ p \in \mathcal{P}}}\left(1-\frac{\rho_{2}(p)}{p^{2}}\right)=\frac{\left(c_{2}(f, q) A_{1}+o(1)\right)}{(\eta \log N)^{\frac{2}{q-1}}} .
$$

The contribution to our upper bound from the $\eta, \eta^{\prime}$ is then

$$
\frac{1}{\eta^{\frac{q-2}{q-1}} \eta^{\frac{2}{q-1}}}
$$

Therefore, to give an optimal result, we want to maximise

$$
\eta^{\prime q-2} \eta^{2}
$$

subject to the constraints

$$
\eta \leq 1-\delta
$$


and

$$
\eta+\eta^{\prime} \leq 2-\frac{\log R_{1}}{\log N}-\delta
$$

By monotonicity it is clear that the maximum occurs when we have equality in the last constraint so

$$
\eta^{\prime}=2-\delta-\eta-\frac{\log R_{1}}{\log N}
$$

We therefore wish to maximise

$$
\left(2-\delta-\eta-\frac{\log R_{1}}{\log N}\right)^{q-2} \eta^{2}
$$

for $\eta \in(0,1-\delta]$. Taking logs we maximise

$$
(q-2) \log \left(2-\delta-\eta-\frac{\log R_{1}}{\log N}\right)+2 \log \eta
$$

so we solve

$$
-(q-2)\left(2-\delta-\eta-\frac{\log R_{1}}{\log N}\right)^{-1}+2 \eta^{-1}=0
$$

This gives

$$
\eta=2 q^{-1}\left(2-\delta-\frac{\log R_{1}}{\log N}\right)
$$

Observe that this is in $(0,1-\delta]$ if $q \geq 5$ and $\delta$ is sufficiently small. We then get

$$
\eta^{\prime}=2-\delta-\eta-\frac{\log R_{1}}{\log N}=\left(1-2 q^{-1}\right)\left(2-\delta-\frac{\log R_{1}}{\log N}\right) .
$$

If $q \geq 5$ and $\delta$ is sufficiently small then $\eta^{\prime}>0$. The factor coming from $\eta, \eta^{\prime}$ is thus

$$
\left(\left(1-2 q^{-1}\right)\left(2-\delta-\frac{\log R_{1}}{\log N}\right)\right)^{-\frac{q-2}{q-1}}\left(2 q^{-1}\left(2-\delta-\frac{\log R_{1}}{\log N}\right)\right)^{-\frac{2}{q-1}} .
$$

This increases as we increase $R_{1}$ so we can replace $R_{1}$ by $r$ getting the smooth weight

$$
w(r, \delta)=\left(1-2 q^{-1}\right)^{-\frac{q-2}{q-1}}\left(2 q^{-1}\right)^{-\frac{2}{q-1}}\left(2-\delta-\frac{\log R_{1}}{\log N}\right)^{-\frac{q}{q-1}} .
$$

Combining all of the above we see that the main term in our estimate for $S_{4}$ is

$$
\frac{A_{1} A_{2} c_{1}(f, q) c_{2}(f, q) N^{2}}{\Delta^{2}(\log N)^{\frac{q}{q-1}}} \sum_{\substack{r \leq R /\left(f_{0} ; \Delta\right) \\ r \equiv r_{0}(\bmod q)}} w(r, \delta) g(r)
$$

where $g(r)$ is the multiplicative function which is 0 unless all the prime factors of $r$ are in $\mathcal{P}^{\prime}$, in which case it is given by

$$
g(r)=\frac{\rho_{1}(r)}{r^{2}} \prod_{p \mid r}\left(1-\frac{\rho_{1}(p)}{p^{2}}\right)^{-1} .
$$

To estimate the sum over $r$ we begin by dealing with the $r$ which are squarefree. 
Lemma 6.1. The multiplicative function $g$, when restricted to squarefree numbers, satisfies all the hypotheses of Lemma 5.1.

Proof. Since $q \mid \Delta$ we know that $g(q)=0$. In addition if $g(p) \neq 0$ then $p \in \mathcal{P}^{\prime}$ so $p \not \equiv 1$ $(\bmod q)$.

If $a \not \equiv 0,1(\bmod q)$ then

$$
\begin{aligned}
\sum_{\substack{p \leq x \\
p \equiv a(\bmod q)}} g(p) \log p & =\sum_{\substack{p \leq x \\
p \equiv a)(\bmod q)}}\left(1-\frac{\rho_{1}(p)}{p^{2}}\right)^{-1} \frac{\rho_{1}(p)}{p^{2}} \log p+O(1) \\
& =\sum_{\substack{p \leq x \\
p \equiv a{ }_{(\bmod q)}}} \frac{\rho_{1}(p)}{p^{2}} \log p+O(1) \\
& =\sum_{p \equiv a{ }_{p \leq x}} \frac{(p-1) \nu(p)+1}{p^{2}} \log p+O(1) \\
& =\frac{1}{q-1} \log x+O(1)
\end{aligned}
$$

This establishes (15) and (9) with

$$
k=\frac{q-2}{q-1} .
$$

If $2 \leq w<z$ then, by Lemma 4.2, we have

$$
\prod_{w \leq p<z}(1+g(p))=\prod_{\substack{w \leq p<z \\ p \in \mathcal{P}^{\prime}}}\left(1-\frac{\rho_{1}(p)}{p^{2}}\right)^{-1} \ll\left(\frac{\log z}{\log w}\right)^{\frac{q-2}{q-1}}
$$

so (6) holds. Finally

$$
\sum_{p} g(p)^{2} \log p=\sum_{p \in \mathcal{P}^{\prime}}\left(1-\frac{\rho_{1}(p)}{p^{2}}\right)^{-2} \frac{\rho_{1}(p)^{2}}{p^{4}} \log p \ll \sum_{p} \frac{1}{p^{2}} \log p<\infty
$$

and therefore (77) also holds. 
Summing by parts and applying Lemma 5.1 we have

$$
\begin{aligned}
& \sum_{\substack{r \leq R /\left(f_{0} ; \Delta\right) \\
\mu(r) \neq 0, r \equiv r_{0}(\bmod q)}} w(r, \delta) g(r) \\
& \leq \sum_{\substack{r \leq R \\
\mu(r) \neq 0, r \equiv r_{0}}} w(\bmod q) \\
& =w(R, \delta) \sum_{\substack{r \leq R \\
\mu(r) \neq 0, r \equiv r_{0}(\bmod q)}} g(r)-\int_{1}^{R}\left(\sum_{\substack{r \leq t \\
\mu(r) \neq 0, r \equiv r_{0}(\bmod q)}} g(r)\right) w^{\prime}(t) d t \\
& =\left(c_{g}+o(1)\right) \frac{1}{q-1}\left(w(R, \delta)(\log R)^{\frac{q-2}{q-1}}-\int_{1}^{R} w^{\prime}(t)(\log t)^{\frac{q-2}{q-1}} d t\right) \\
& =\left(c_{g}+o(1)\right) \frac{q-2}{(q-1)^{2}} \int_{1}^{R} w(t, \delta)(\log t)^{\frac{-1}{q-1}} t^{-1} d t \\
& =\left(c_{g}+o(1)\right) \frac{q-2}{(q-1)^{2}}(\log N)^{\frac{q-2}{q-1}} \int_{0}^{\log R / \log N} w\left(N^{s}, \delta\right) s^{\frac{-1}{q-1}} d s,
\end{aligned}
$$

where

$$
c_{g}=\frac{1}{\Gamma\left(2-\frac{1}{q-1}\right)} \prod_{p}\left(1-\frac{1}{p}\right)^{\frac{q-2}{q-1}}(1+g(p)) .
$$

Observe that

$$
w\left(N^{s}, \delta\right)=\left(1-2 q^{-1}\right)^{-\frac{q-2}{q-1}}\left(2 q^{-1}\right)^{-\frac{2}{q-1}}(2-\delta-s)^{-\frac{q}{q-1}}
$$

does not depend on $N$. In addition

$$
\frac{\log R}{\log N}=\frac{\log x}{\log N}-\beta=3-\beta+o(1)
$$

as $N \rightarrow \infty$. We may therefore replace the upper limit of integration by $3-\beta$ at the cost of an error which is $o(1)$. We conclude that

$$
\sum_{\substack{r \leq R /\left(f_{0} ; \Delta\right) \\ \mu(r) \neq 0, r \equiv r_{0}(\bmod q)}} w(r, \delta) g(r) \leq\left(c_{g}+o(1)\right) \frac{q-2}{(q-1)^{2}}(\log N)^{\frac{q-2}{q-1}} \int_{0}^{3-\beta} w\left(N^{s}, \delta\right) s^{\frac{-1}{q-1}} d s .
$$

Let

$$
W(s)=w\left(N^{s}, 0\right) s^{\frac{-1}{q-1}} .
$$

For any $\epsilon>0$ we can choose a sufficiently small $\delta$ to get

$$
\int_{0}^{3-\beta} w\left(N^{s}, \delta\right) s^{\frac{-1}{q-1}} d s \leq \int_{0}^{3-\beta} W(s) d s+\epsilon+o(1)
$$


and thus

$$
\sum_{\substack{r \leq R /\left(f_{0} ; \Delta\right) \\ \mu(r) \neq 0, r \equiv r_{0}(\bmod q)}} w(r, \delta) g(r) \leq\left(c_{g}+\epsilon+o(1)\right) \frac{q-2}{(q-1)^{2}}(\log N)^{\frac{q-2}{q-1}} \int_{0}^{3-\beta} W(s) d s .
$$

It remains to deal with the sum over those $r$ which are not squarefree.

Lemma 6.2. For any $\epsilon>0$ there exists a $P_{1}$, depending on $\epsilon, q$ and $f$ but not on $N$, such that if we include all primes $p \leq P_{1}$ in $\mathcal{P}_{1}$ then

$$
\sum_{\substack{r \leq R /\left(f_{0} ; \Delta\right) \\ \mu(r)=0, r \equiv r_{0}(\bmod q)}} w(r, \delta) g(r) \leq(\epsilon+o(1))(\log N)^{\frac{q-2}{q-1}} .
$$

Proof. Any $r \in \mathbb{N}$ can be written uniquely as $r=r_{1} r_{2}$ for some squarefree $r_{1}$ and some squarefull $r_{2}$ satisfying $\left(r_{1} ; r_{2}\right)=1$. In addition if $\mu(r)=0$ then $r_{2}>1$. Since $w(r, \delta) \ll 1$ and $g(r) \geq 0$ our sum may be bounded by

$$
\sum_{\substack{r_{1} r_{2} \leq R /\left(f_{0} ; \Delta\right) \\ r_{2}>1}} g\left(r_{1}\right) g\left(r_{2}\right)
$$

where the sum is restricted to squarefree $r_{1}$ and squarefull $r_{2}$ with $\left(r_{1} ; r_{2}\right)=1$.

Since $g(r)$ is supported on numbers having no prime factor in $\mathcal{P}_{1}$ we can use Lemma 4.3 to deduce that for all $r$

$$
g(r) \ll_{\epsilon} r^{-\frac{2}{3}+\epsilon} .
$$

It follows that

$$
\sum_{r \text { squarefull }} g(r)<\infty
$$

Furthermore, if we include all primes up to $P_{1}$ in $\mathcal{P}_{1}$ then all terms in this sum with $r \leq P_{1}$ are 0 . It follows that for any $\epsilon>0$ we can choose $P_{1}$ sufficiently large so that

$$
\sum_{\substack{r>1 \\ r \text { squarefull }}} g(r)<\epsilon
$$

Our original sum may therefore be bounded by

$$
\left(\sum_{\substack{r_{1} \leq R /\left(f_{0} ; \Delta\right) \\ \mu\left(r_{1}\right) \neq 0}} g\left(r_{1}\right)\right)\left(\sum_{r_{2} \text { squarefull }} g\left(r_{2}\right)\right) .
$$

Using Lemma 5.1 the first sum is $O\left((\log R)^{\frac{q-2}{q-1}}\right)$ so the result follows. 
It follows from the last lemma that, with a suitable choice of $P_{1}$, the non-squarefree $r$ give a contribution to $S_{4}$ bounded by

$$
\frac{(\epsilon+o(1))}{(\log N)^{\frac{2}{q-1}}}
$$

Combining all of the results of this subsection we see that for any $\epsilon>0$, by taking sufficiently many small primes in $\mathcal{P}_{1}$ and $\delta$ sufficiently small, we get the bound

$$
S_{4} \leq \int_{0}^{3-\beta} W(s) d s \frac{\left(A_{1} A_{2} c_{g} c_{1}(f, q) c_{2}(f, q)+\epsilon+o(1)\right)(q-2) N^{2}}{\Delta^{2}(q-1)^{2}(\log N)^{\frac{2}{q-1}}} .
$$

Finally we must remove the constants $c_{g}, c_{1}(f, q)$ from this bound. Recall that these are defined by

$$
\begin{aligned}
c_{g} & =\frac{1}{\Gamma\left(2-\frac{1}{q-1}\right)} \prod_{p}\left(1-\frac{1}{p}\right)^{\frac{q-2}{q-1}}(1+g(p)) \\
& =\frac{1}{\Gamma\left(2-\frac{1}{q-1}\right)} \lim _{x \rightarrow \infty} \prod_{p \leq x}\left(1-\frac{1}{p}\right)^{\frac{q-2}{q-1}}(1+g(p)) \\
& =\frac{1}{\Gamma\left(2-\frac{1}{q-1}\right)} \lim _{x \rightarrow \infty}\left(\frac{e^{-\gamma}}{\log x}\right)^{\frac{q-2}{q-1}} \prod_{\substack{p \leq x \\
p \in \mathcal{P}^{\prime}}}\left(1-\frac{\rho_{1}(p)}{p^{2}}\right)^{-1}
\end{aligned}
$$

and

$$
c_{1}(f, q)=\lim _{x \rightarrow \infty}(\log x)^{\frac{q-2}{q-1}} \prod_{\substack{p<x \\ p \in \mathcal{P}^{\prime}}}\left(1-\frac{\rho_{1}(p)}{p^{2}}\right) .
$$

It follows that

$$
c_{g} c_{1}(f, q)=\frac{e^{-\gamma \frac{q-2}{q-1}}}{\Gamma\left(2-\frac{1}{q-1}\right)} .
$$

We therefore conclude that

$$
S_{4} \leq \int_{0}^{3-\beta} W(s) d s \frac{\left(A_{1} A_{2} e^{-\gamma \frac{q-2}{q-1}} c_{2}(f, q)(q-2)+\epsilon+o(1)\right) N^{2}}{\Delta^{2} \Gamma\left(2-\frac{1}{q-1}\right)(q-1)^{2}(\log N)^{\frac{2}{q-1}}} .
$$

\subsection{Conclusion}

Combining the bounds for $S_{1}, S_{2}, S_{3}$ and $S_{4}$ we conclude that for any $\epsilon>0$ we can take sufficiently many small primes in $\mathcal{P}_{1}$ so that we have, as $N \rightarrow \infty$, that

$$
S(\mathcal{A}, \mathcal{P}, x) \geq \frac{c_{2}(f, q) N^{2}}{\Delta^{2}(\log N)^{\frac{2}{q-1}}}(F(q)-\epsilon+o(1))
$$


where

$$
F(q)=B_{1}-\frac{A_{1}}{q-1} \int_{1}^{\beta}(2-s)^{-\frac{2}{q-1}} \frac{d s}{s}-\frac{A_{1} A_{2} e^{-\gamma \frac{q-2}{q-1}}(q-2)}{\Gamma\left(2-\frac{1}{q-1}\right)(q-1)^{2}} \int_{0}^{3-\beta} W(s) d s
$$

and

$$
W(s)=\left(1-2 q^{-1}\right)^{-\frac{q-2}{q-1}}\left(2 q^{-1}\right)^{-\frac{2}{q-1}}(2-s)^{-\frac{q}{q-1}} s^{\frac{-1}{q-1}} .
$$

Recall that the values $A_{1}, B_{1}$ and $A_{2}$ all depend on $q$. As the sieve dimension $\kappa \rightarrow 0$ we have $A(\kappa), B(\kappa) \rightarrow 1$. It follows that

$$
\lim _{q \rightarrow \infty} F(q)=1
$$

Therefore $F(q)$ is positive for $q \geq q_{0}$ for some absolute $q_{0}$. For any such $q$ we can then choose $N$ sufficiently large to get $S(\mathcal{A}, \mathcal{P}, x)>0$ and thus (11) has a rational solution.

To give the best possible bound we must choose $\beta$ to minimise

$$
\int_{1}^{\beta}(2-s)^{-\frac{2}{q-1}} \frac{d s}{s}+\frac{A_{2} e^{-\gamma \frac{q-2}{q-1}}(q-2)}{\Gamma\left(2-\frac{1}{q-1}\right)(q-1)} \int_{0}^{3-\beta} W(s) d s .
$$

Thus we must solve

$$
(2-\beta)^{-\frac{2}{q-1}} \beta^{-1}-\frac{A_{2} e^{-\gamma \frac{q-2}{q-1}}(q-2)}{\Gamma\left(2-\frac{1}{q-1}\right)(q-1)} W(3-\beta)=0
$$

that is

$$
(2-\beta)^{-\frac{2}{q-1}} \beta^{-1}-\frac{A_{2} e^{-\gamma \frac{q-2}{q-1}}(q-2)}{\Gamma\left(2-\frac{1}{q-1}\right)(q-1)}\left(1-2 q^{-1}\right)^{-\frac{q-2}{q-1}}\left(2 q^{-1}\right)^{-\frac{2}{q-1}}(\beta-1)^{-\frac{q}{q-1}}(3-\beta)^{\frac{-1}{q-1}}=0 .
$$

To complete the proof of Theorem 1.1 we must show that for all primes $q \geq 7$ there exists a choice of $\beta \in\left(\frac{3}{2}, 2\right)$ for which $F(q)>0$. The case $q=7$ is the most delicate numerically so we deal with it first.

From Friedlander and Iwaniec's table in [8, Section 11.19] we obtain the value

$$
A_{2}=A(5 / 6)=2.56140 \ldots
$$

The constants $A_{1}, B_{1}$ are given by [8, (11.62)]. We find by numerical integration that

$$
A_{1}=A(1 / 3)=1.27713 \ldots
$$

and

$$
B 1=B(1 / 3)=0.71213 \ldots
$$

By solving the above equation numerically we discover that the optimal choice for $\beta$ is approximately 1.994. We conclude, evaluating all integral numerically, that

$$
F(7) \approx 0.0504>0
$$


Due to the use of numerical integration we cannot be completely sure that $F(7)>0$. However we are confident that the computations were sufficiently accurate to make this extremely likely.

For $q \geq 11$ we do not need to be quite so careful. Since $A_{2} \leq A(1)$ we have

$$
F(q) \geq B_{1}-\frac{A_{1}}{q-1} \int_{1}^{\beta}(2-s)^{-\frac{2}{q-1}} \frac{d s}{s}-\frac{A_{1} A(1) e^{-\gamma \frac{q-2}{q-1}}(q-2)}{\Gamma\left(2-\frac{1}{q-1}\right)(q-1)^{2}} \int_{0}^{3-\beta} W(s) d s .
$$

As $q$ increases $B_{1}=B\left(\frac{2}{q-1}\right)$ is increasing whereas $A_{1}=A\left(\frac{2}{q-1}\right)$ is decreasing. In addition, for any $s \in(1, \beta)$ the quantity

$$
(2-s)^{-\frac{2}{q-1}}
$$

is decreasing, as are

$$
\begin{gathered}
e^{-\gamma \frac{q-2}{q-1}} \\
\frac{q-2}{(q-1)^{2}}
\end{gathered}
$$

and

$$
\frac{1}{\Gamma\left(2-\frac{1}{q-1}\right)}
$$

Recall that

$$
W(s)=\left(1-2 q^{-1}\right)^{-\frac{q-2}{q-1}}\left(2 q^{-1}\right)^{-\frac{2}{q-1}}(2-s)^{-\frac{q}{q-1}} s^{\frac{-1}{q-1}} .
$$

It can be shown that for any $s \in(0,3-\beta)$ this decreases as we increase $q$.

We can conclude that, for a fixed $\beta \in\left(\frac{3}{2}, 2\right)$, the above bound for $F(q)$ is an increasing function of $q$. It follows that it is sufficient that the bound is positive when $q=11$. Using that

$$
\begin{gathered}
A(1)=2 e^{\gamma}=3.562144 \ldots, \\
A(1 / 5)=1.15147 \ldots
\end{gathered}
$$

and

$$
B(1 / 5)=0.92055 \ldots
$$

we can deduce, by taking $\beta=1.9$, that for any prime $q \geq 11$ we have

$$
F(q) \geq 0.514
$$

In conclusion, $F(q)>0$ for all primes $q \geq 7$ so Theorem 1.1 holds for all primes $q \geq 7$. 


\section{References}

[1] H.-J. Bartels. Zur Arithmetik von Konjugationsklassen in algebraischen Gruppen. J. Algebra, 70(1):179-199, 1981.

[2] T. D. Browning and D. R. Heath-Brown. Quadratic polynomials represented by norm forms. Geom. Funct. Anal., 22(5):1124-1190, 2012.

[3] T. D. Browning and L. Matthiesen. Norm forms for arbitrary number fields as products of linear polynomials. arXiv:1307.7641.

[4] J.-L. Colliot-Thélène and P. Salberger. Arithmetic on some singular cubic hypersurfaces. Proc. London Math. Soc. (3), 58(3):519-549, 1989.

[5] J.-L. Colliot-Thélène, J.-J. Sansuc, and P. Swinnerton-Dyer. Intersections of two quadrics and Châtelet surfaces. I. J. Reine Angew. Math., 373:37-107, 1987.

[6] S. Daniel. On the divisor-sum problem for binary forms. J. Reine Angew. Math., 507:107129, 1999.

[7] U. Derenthal, A. Smeets, and D. Wei. Universal torsors and values of quadratic polynomials represented by norms. arXiv:1202.3567.

[8] J. Friedlander and H. Iwaniec. Opera de cribro, volume 57 of American Mathematical Society Colloquium Publications. American Mathematical Society, Providence, RI, 2010.

[9] G. Gras. Class field theory. Springer Monographs in Mathematics. Springer-Verlag, Berlin, 2003. From theory to practice, Translated from the French manuscript by Henri Cohen.

Mathematical Institute, University of Oxford, Andrew Wiles Building, Radcliffe Observatory Quarter, Woodstock Road,

Oxford

OX2 6GG

UK

irving@maths.ox.ac.uk 